\title{
Analytical Data Report of Water Samples Collected For I-129 Analysis
}

Michael Lindberg

October 2009

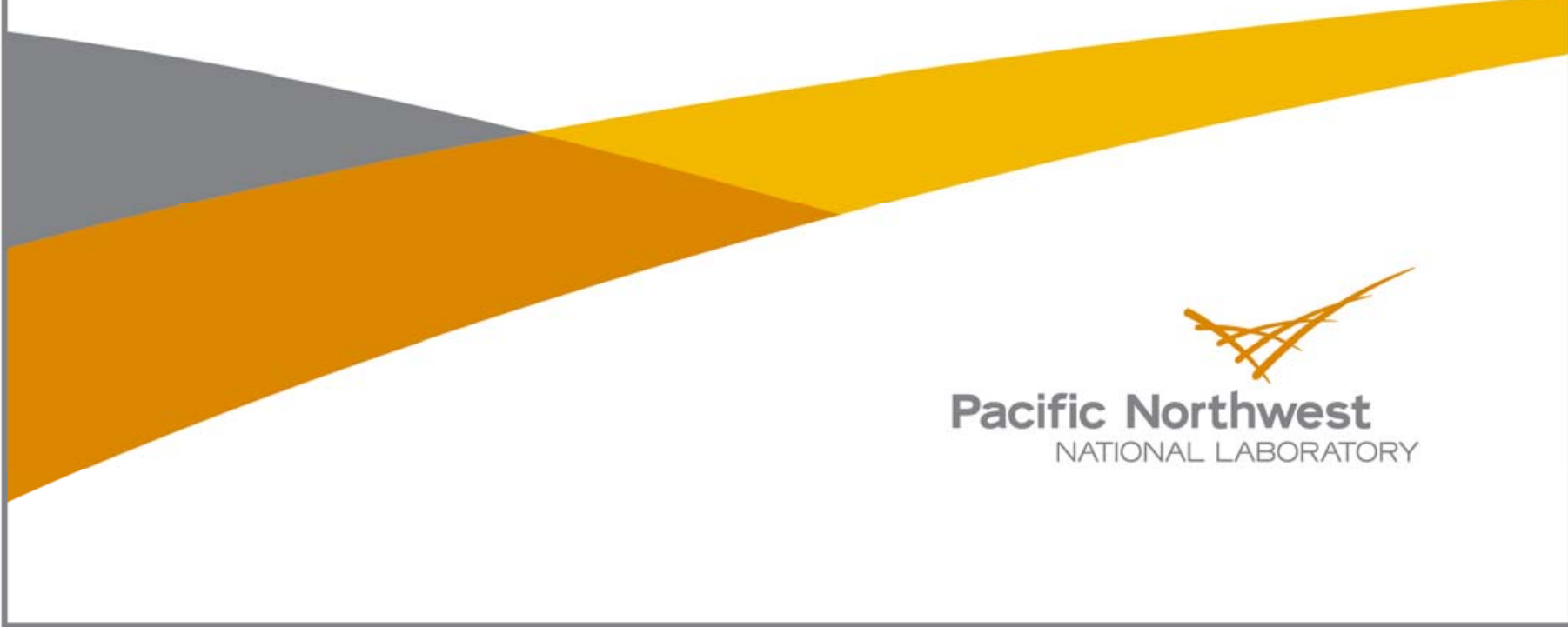




\title{
DISCLAIMER
}

This report was prepared as an account of work sponsored by an agency of the United States Government. Neither the United States Government nor any agency thereof, nor Battelle Memorial Institute, nor any of their employees, makes any warranty, express or implied, or assumes any legal liability or responsibility for the accuracy, completeness, or usefulness of any information, apparatus, product, or process disclosed, or represents that its use would not infringe privately owned rights. Reference herein to any specific commercial product, process, or service by trade name, trademark, manufacturer, or otherwise does not necessarily constitute or imply its endorsement, recommendation, or favoring by the United States Government or any agency thereof, or Battelle Memorial Institute. The views and opinions of authors expressed herein do not necessarily state or reflect those of the United States Government or any agency thereof.

\author{
PACIFIC NORTHWEST NATIONAL LABORATORY \\ operated by \\ BATTELLE \\ for the \\ UNITED STATES DEPARTMENT OF ENERGY \\ under Contract DE-AC05-76RL01830
}

Printed in the United States of America
Available to DOE and DOE contractors from the Office of Scientific and Technical Information,
P.O. Box 62, Oak Ridge, TN 37831-0062;
ph: (865) 576-8401
fax: $(865)$ 576-5728
email: reports@adonis.osti.gov

\begin{abstract}
Available to the public from the National Technical Information Service, U.S. Department of Commerce, 5285 Port Royal Rd., Springfield, VA 22161 ph: (800) 553-6847 fax: $(703) 605-6900$ email: orders@ntis.fedworld.gov online ordering: http://www.ntis.gov/ordering.htm
\end{abstract}

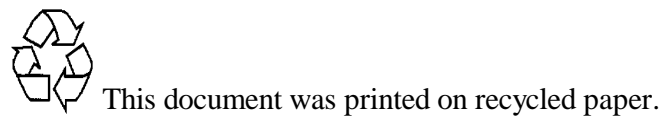




\section{Analytical Data Report of Water Samples Collected For I-129 Analysis}

M Lindberg

October 2009

Prepared for the U.S. Department of Energy

under Contract DE-AC05-76RL01830

Pacific Northwest National Laboratory

Richland, Washington 99352 
10/19/09 11:20

To: Dale Dyekman

From: Michael J. Lindberg

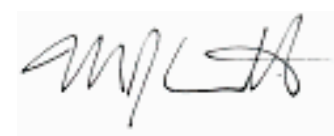

Environmental Sciences Laboratory

Energy and Environment Directorate, Pacific Northwest National Laboratory

Subject: Analytical Data Report for Ground Water Samples Collected for I-129 Groundwater, Sample Delivery Group ESL090004, SAF Number S09-004

This letter contains the following information for sample delivery group ESL090004

- $\quad$ Cover Sheet

- Narrative

- Analytical Results

- Chain of Custodies 


\section{Introduction}

Between April 14, 2009 and August 25, 2009 groundwater samples were received for I-129 analysis.

\section{Analytical Results/Methodology}

The analyses for this project were performed at the 325 building located in the 300 Area of the Hanford Site. The analyses were performed according to Pacific Northwest National Laboratory (PNNL) approved procedures and/or nationally recognized test procedures. The data sets include the sample identification numbers, analytical results, estimated quantification limits (EQL), and quality control data.

\section{Quality Control}

The preparatory and analytical quality control requirements, calibration requirements, acceptance criteria, and failure actions are defined in the on-line QA plan “Conducting Analytical Work in Support of Regulatory Programs” (CAW). This QA plan implements the Hanford Analytical Services Quality Assurance Requirements Documents (HASQARD) for PNNL.

\section{Definitions}

Dup Duplicate

RPD Relative Percent Difference

NR No Recovery (percent recovery less than zero)

ND Non-Detectable

$\%$ REC Percent Recovery

\section{Sample Receipt}

Samples were received with a chain of custody (COC) and were analyzed according to the sample identification numbers supplied by the client. All samples were refrigerated upon receipt until prepared for analysis.

All samples were received with custody seals intact unless noted in the Case Narrative.

\section{Holding Times}

Holding time is defined as the time from sampling to the time of analyses. The prescribed holding times were met for all analytes unless noted in the Case Narrative.

\section{Analytical Results}

All reported analytical results meet the requirements of the CAW or client specified SOW unless noted in the case narrative. 


\section{Case Narrative Report}

\section{Hold Time:}

No discrepancies noted.

\section{Preparation Blank (PB):}

No preparation was performed therefore no blanks were analyzed.

\section{Duplicate (DUP):}

No preparation was performed therefore no duplicates were analyzed.

\section{Laboratory Control Samples (LCS):}

No preparation was performed therefore no LCSs were analyzed.

\section{Post Spike (PS):}

No preparation was performed therefore no PSs were analyzed.

\section{Matrix Spike (MS):}

Not applicable.

\section{Other QC Criteria:}

None noted.

\section{DISCLAIMER}

This report was prepared as an account of work sponsored by an agency of the United States Government. Neither the United States Government nor any agency thereof, nor Battelle Memorial Institute, nor any of their employees, makes any warranty, express or implied, or assumes any legal liability or responsibility for the accuracy, completeness, or usefulness of any information, apparatus, product, or process disclosed, or represents that its use would not infringe privately owned rights. Reference herein to any specific commercial product, process, or service by trade name, trademark, manufacturer, or otherwise does not necessarily constitute or imply its endorsement, recommendation, or favoring by the United States Government or any agency thereof, or Battelle Memorial Institute. The views and opinions of authors expressed herein do not necessarily state or reflect those of the United States Government or any agency thereof. 


\section{I-129 Groundwaters}

$\begin{array}{lll}\text { HEIS No. } & \text { Laboratory ID } & \text { Matrix } \\ \text { B203D8 } & 0905001-01 & \text { WATER } \\ \text { B203F3 } & 0905001-02 & \text { WATER } \\ \text { B203F8 } & 0905001-03 & \text { WATER } \\ \text { B203M8 } & 0905001-04 & \text { WATER } \\ \text { B203P9 } & 0905001-05 & \text { WATER } \\ \text { B203R4 } & 0905001-06 & \text { WATER } \\ \text { B203V1 } & 0905001-07 & \text { WATER } \\ \text { B203V5 } & 0905001-08 & \text { WATER } \\ \text { B203H3 } & 0905001-09 & \text { WATER } \\ \text { B202Y0 } & 0905001-10 & \text { WATER } \\ \text { B20979 } & 0905001-11 & \text { WATER } \\ \text { B20980 } & 0905001-12 & \text { WATER } \\ \text { B20981 } & 0905001-13 & \text { WATER } \\ \text { B20982 } & 0905001-14 & \text { WATER } \\ \text { B20983 } & 0905001-15 & \text { WATER } \\ \text { B20984 } & 0905001-16 & \text { WATER } \\ \text { B20LP2 } & 0905001-17 & \text { WATER } \\ \text { B20LR4 } & 0905001-18 & \text { WATER } \\ \text { B20LT1 } & 0905001-19 & \text { WATER } \\ \text { B20LT4 } & 0905001-20 & \text { WATER } \\ \text { B20MX3 } & 0905001-21 & \text { WATER } \\ \text { B20PJ5 } & 0905001-22 & \text { WATER } \\ \text { B20PP7 } & 0905001-23 & \text { WATER } \\ \text { B20P35 } & 0905001-24 & \text { WATER } \\ \text { B20NX6 } & 0905001-25 & \text { WATER } \\ \text { B20MY5 } & 0905001-26 & \text { WATER }\end{array}$

\begin{tabular}{llll} 
Date Collected & \multicolumn{2}{c}{ Date Received } \\
4/29/09 & $09: 55$ & $4 / 30 / 09$ & $11: 45$ \\
4/29/09 & $08: 47$ & $4 / 30 / 09$ & $11: 45$ \\
4/29/09 & $10: 53$ & $4 / 30 / 09$ & $11: 45$ \\
4/26/09 & $09: 18$ & $4 / 23 / 09$ & $08: 50$ \\
4/10/09 & $08: 40$ & $4 / 14 / 09$ & $14: 15$ \\
4/20/09 & $12: 04$ & $4 / 23 / 09$ & $08: 50$ \\
4/20/09 & $13: 31$ & $4 / 23 / 09$ & $08: 50$ \\
4/20/09 & $12: 31$ & $4 / 23 / 09$ & $08: 50$ \\
4/30/09 & $09: 39$ & $5 / 4 / 09$ & $13: 40$ \\
4/29/09 & $10: 50$ & $4 / 30 / 09$ & $11: 45$ \\
$6 / 16 / 09$ & $10: 30$ & $6 / 17 / 09$ & $11: 30$ \\
$6 / 16 / 09$ & $10: 30$ & $6 / 17 / 09$ & $11: 30$ \\
$6 / 16 / 09$ & $10: 30$ & $6 / 17 / 09$ & $11: 30$ \\
$6 / 16 / 09$ & $10: 30$ & $6 / 17 / 09$ & $11: 30$ \\
$6 / 16 / 09$ & $10: 30$ & $6 / 17 / 09$ & $11: 30$ \\
$6 / 16 / 09$ & $10: 30$ & $6 / 17 / 09$ & $11: 30$ \\
$6 / 23 / 09$ & $13: 38$ & $6 / 24 / 09$ & $10: 28$ \\
$6 / 23 / 09$ & $12: 44$ & $6 / 24 / 09$ & $10: 28$ \\
$6 / 23 / 09$ & $11: 30$ & $6 / 24 / 09$ & $10: 28$ \\
$6 / 23 / 09$ & $10: 00$ & $6 / 24 / 09$ & $10: 28$ \\
$6 / 17 / 09$ & $10: 35$ & $6 / 23 / 09$ & $12: 47$ \\
$6 / 24 / 09$ & $10: 45$ & $6 / 25 / 09$ & $12: 00$ \\
$7 / 8 / 09$ & $09: 38$ & $7 / 14 / 09$ & $13: 00$ \\
$6 / 26 / 09$ & $11: 26$ & $6 / 29 / 09$ & $09: 30$ \\
$6 / 25 / 09$ & $12: 30$ & $6 / 29 / 09$ & $09: 30$ \\
$8 / 14 / 09$ & $11: 00$ & $8 / 25 / 09$ & $13: 00$
\end{tabular}


The following analyses were performed on the following samples included in this report:

Iodine 129 Water by ICPMS

SAMPLES ANALYZED IN THIS REPORT

\begin{tabular}{|c|c|c|}
\hline $\begin{array}{l}\text { HEIS No. } \\
\text { B203D8 }\end{array}$ & $\begin{array}{l}\text { Laboratory ID } \\
0905001-01\end{array}$ & $\begin{array}{l}\text { Matrix } \\
\text { WATER }\end{array}$ \\
\hline B203F3 & 0905001-02 & WATER \\
\hline B203F8 & 0905001-03 & WATER \\
\hline B203M8 & 0905001-04 & WATER \\
\hline В203P9 & 0905001-05 & WATER \\
\hline B203R4 & 0905001-06 & WATER \\
\hline B203V1 & 0905001-07 & WATER \\
\hline B203V5 & 0905001-08 & WATER \\
\hline В203H3 & 0905001-09 & WATER \\
\hline B202Y0 & 0905001-10 & WATER \\
\hline B20979 & 0905001-11 & WATER \\
\hline B20980 & 0905001-12 & WATER \\
\hline B20981 & 0905001-13 & WATER \\
\hline B20982 & 0905001-14 & WATER \\
\hline B20983 & 0905001-15 & WATER \\
\hline B20984 & 0905001-16 & WATER \\
\hline B20LP2 & 0905001-17 & WATER \\
\hline B20LR4 & 0905001-18 & WATER \\
\hline B20LT1 & 0905001-19 & WATER \\
\hline B20LT4 & 0905001-20 & WATER \\
\hline B20MX3 & 0905001-21 & WATER \\
\hline B20PJ5 & 0905001-22 & WATER \\
\hline В20РP7 & $0905001-23$ & WATER \\
\hline B20P35 & 0905001-24 & WATER \\
\hline B20NX6 & $0905001-25$ & WATER \\
\hline B20MY5 & $0905001-26$ & WATER \\
\hline
\end{tabular}

\begin{tabular}{llll} 
Date Collected & \multicolumn{3}{l}{ Date Received } \\
4/29/09 & $09: 55$ & $4 / 30 / 09$ & $11: 45$ \\
4/29/09 & $08: 47$ & $4 / 30 / 09$ & $11: 45$ \\
4/29/09 & $10: 53$ & $4 / 30 / 09$ & $11: 45$ \\
4/26/09 & $09: 18$ & $4 / 23 / 09$ & $08: 50$ \\
4/10/09 & $08: 40$ & $4 / 14 / 09$ & $14: 15$ \\
4/20/09 & $12: 04$ & $4 / 23 / 09$ & $08: 50$ \\
$4 / 20 / 09$ & $13: 31$ & $4 / 23 / 09$ & $08: 50$ \\
$4 / 20 / 09$ & $12: 31$ & $4 / 23 / 09$ & $08: 50$ \\
$4 / 30 / 09$ & $09: 39$ & $5 / 4 / 09$ & $13: 40$ \\
$4 / 29 / 09$ & $10: 50$ & $4 / 30 / 09$ & $11: 45$ \\
$6 / 16 / 09$ & $10: 30$ & $6 / 17 / 09$ & $11: 30$ \\
$6 / 16 / 09$ & $10: 30$ & $6 / 17 / 09$ & $11: 30$ \\
$6 / 16 / 09$ & $10: 30$ & $6 / 17 / 09$ & $11: 30$ \\
$6 / 16 / 09$ & $10: 30$ & $6 / 17 / 09$ & $11: 30$ \\
$6 / 16 / 09$ & $10: 30$ & $6 / 17 / 09$ & $11: 30$ \\
$6 / 16 / 09$ & $10: 30$ & $6 / 17 / 09$ & $11: 30$ \\
$6 / 23 / 09$ & $13: 38$ & $6 / 24 / 09$ & $10: 28$ \\
$6 / 23 / 09$ & $12: 44$ & $6 / 24 / 09$ & $10: 28$ \\
$6 / 23 / 09$ & $11: 30$ & $6 / 24 / 09$ & $10: 28$ \\
$6 / 23 / 09$ & $10: 00$ & $6 / 24 / 09$ & $10: 28$ \\
$6 / 17 / 09$ & $10: 35$ & $6 / 23 / 09$ & $12: 47$ \\
$6 / 24 / 09$ & $10: 45$ & $6 / 25 / 09$ & $12: 00$ \\
$7 / 8 / 09$ & $09: 38$ & $7 / 14 / 09$ & $13: 00$ \\
$6 / 26 / 09$ & $11: 26$ & $6 / 29 / 09$ & $09: 30$ \\
$6 / 25 / 09$ & $12: 30$ & $6 / 29 / 09$ & $09: 30$ \\
$8 / 14 / 09$ & $11: 00$ & $8 / 25 / 09$ & $13: 00$
\end{tabular}


Radionuclides By ICP-MS

\begin{tabular}{|c|c|c|c|c|c|c|c|}
\hline CAS \# & Analyte & Results & Units & EQL & Analyzed & Batch & Method \\
\hline HEIS No. & B203D8 & \multicolumn{2}{|c|}{ Lab ID: } & 0905001-01 & & & \\
\hline $15046-84-1$ & Iodine-129 & $1.26 \mathrm{E}-2$ & $\mathrm{ug} / \mathrm{L}$ & $1.00 \mathrm{E}-2$ & $9 / 24 / 09$ & 9I14001 & PNNL-AGG-415 \\
\hline HEIS No. & B203F3 & \multicolumn{2}{|c|}{ Lab ID: } & 0905001-02 & & & \\
\hline $15046-84-1$ & Iodine-129 & $<5.05 \mathrm{E}-3$ & $\mathrm{ug} / \mathrm{L}$ & $5.05 \mathrm{E}-3$ & $10 / 06 / 09$ & 9I14001 & PNNL-AGG-415 \\
\hline HEIS No. & B203F8 & \multicolumn{2}{|c|}{ Lab ID: } & 0905001-03 & & & \\
\hline $15046-84-1$ & Iodine-129 & $1.36 \mathrm{E}-2$ & $\mathrm{ug} / \mathrm{L}$ & $1.00 \mathrm{E}-2$ & $9 / 24 / 09$ & 9I14001 & PNNL-AGG-415 \\
\hline HEIS No. & B203M8 & \multicolumn{2}{|c|}{ Lab ID: } & 0905001-04 & & & \\
\hline $15046-84-1$ & Iodine-129 & $1.30 \mathrm{E}-2$ & $\mathrm{ug} / \mathrm{L}$ & $1.00 \mathrm{E}-2$ & 9/24/09 & 9I14001 & PNNL-AGG-415 \\
\hline HEIS No. & B203P9 & \multicolumn{2}{|c|}{ Lab ID: } & 0905001-05 & & & \\
\hline $15046-84-1$ & Iodine-129 & $<5.05 \mathrm{E}-3$ & $\mathrm{ug} / \mathrm{L}$ & $5.05 \mathrm{E}-3$ & $10 / 06 / 09$ & 9I14001 & PNNL-AGG-415 \\
\hline HEIS No. & B203R4 & \multicolumn{2}{|c|}{ Lab ID: } & 0905001-06 & & & \\
\hline $15046-84-1$ & Iodine-129 & $1.14 \mathrm{E}-2$ & $\mathrm{ug} / \mathrm{L}$ & $1.00 \mathrm{E}-2$ & $9 / 24 / 09$ & 9I14001 & PNNL-AGG-415 \\
\hline HEIS No. & B203V1 & \multicolumn{2}{|c|}{ Lab ID: } & 0905001-07 & & & \\
\hline $15046-84-1$ & Iodine-129 & $9.16 \mathrm{E}-3$ & $\mathrm{ug} / \mathrm{L}$ & $5.05 \mathrm{E}-3$ & $10 / 06 / 09$ & 9I14001 & PNNL-AGG-415 \\
\hline HEIS No. & B203V5 & \multicolumn{2}{|c|}{ Lab ID: } & 0905001-08 & & & \\
\hline $15046-84-1$ & Iodine-129 & $1.16 \mathrm{E}-2$ & $\mathrm{ug} / \mathrm{L}$ & $1.00 \mathrm{E}-2$ & $9 / 24 / 09$ & 9I14001 & PNNL-AGG-415 \\
\hline HEIS No. & В203Н3 & \multicolumn{2}{|c|}{ Lab ID: } & 0905001-09 & & & \\
\hline $15046-84-1$ & Iodine-129 & $<5.05 \mathrm{E}-3$ & $\mathrm{ug} / \mathrm{L}$ & $5.05 \mathrm{E}-3$ & $10 / 06 / 09$ & 9I14001 & PNNL-AGG-415 \\
\hline HEIS No. & B202Y0 & \multicolumn{2}{|c|}{ Lab ID: } & 0905001-10 & & & \\
\hline $15046-84-1$ & Iodine-129 & $<5.05 \mathrm{E}-3$ & $\mathrm{ug} / \mathrm{L}$ & $5.05 \mathrm{E}-3$ & $10 / 06 / 09$ & 9I14001 & PNNL-AGG-415 \\
\hline HEIS No. & B20979 & \multicolumn{2}{|c|}{ Lab ID: } & 0905001-11 & & & \\
\hline $15046-84-1$ & Iodine-129 & $3.30 \mathrm{E}-2$ & $\mathrm{ug} / \mathrm{L}$ & $5.05 \mathrm{E}-3$ & $10 / 06 / 09$ & 9I14001 & PNNL-AGG-415 \\
\hline HEIS No. & B20980 & \multicolumn{2}{|c|}{ Lab ID: } & 0905001-12 & & & \\
\hline $15046-84-1$ & Iodine-129 & $4.31 \mathrm{E}-2$ & $\mathrm{ug} / \mathrm{L}$ & $1.00 \mathrm{E}-2$ & $9 / 24 / 09$ & 9I14001 & PNNL-AGG-415 \\
\hline HEIS No. & B20981 & \multicolumn{2}{|c|}{ Lab ID: } & 0905001-13 & & & \\
\hline $15046-84-1$ & Iodine-129 & $3.66 \mathrm{E}-2$ & $\mathrm{ug} / \mathrm{L}$ & $1.00 \mathrm{E}-2$ & $9 / 24 / 09$ & 9I14001 & PNNL-AGG-415 \\
\hline HEIS No. & B20982 & \multicolumn{2}{|c|}{ Lab ID: } & 0905001-14 & & & \\
\hline $15046-84-1$ & Iodine-129 & $<5.05 \mathrm{E}-3$ & $\mathrm{ug} / \mathrm{L}$ & $5.05 \mathrm{E}-3$ & $10 / 06 / 09$ & 9I14001 & PNNL-AGG-415 \\
\hline HEIS No. & B20983 & \multicolumn{2}{|c|}{ Lab ID: } & 0905001-15 & & & \\
\hline $15046-84-1$ & Iodine-129 & $<5.05 \mathrm{E}-3$ & $\mathrm{ug} / \mathrm{L}$ & $5.05 \mathrm{E}-3$ & $10 / 07 / 09$ & 9I14001 & PNNL-AGG-415 \\
\hline HEIS No. & B20984 & \multicolumn{2}{|c|}{ Lab ID: } & 0905001-16 & & & \\
\hline $15046-84-1$ & Iodine-129 & $<5.05 \mathrm{E}-3$ & $\mathrm{ug} / \mathrm{L}$ & $5.05 \mathrm{E}-3$ & $10 / 07 / 09$ & 9I14001 & PNNL-AGG-415 \\
\hline HEIS No. & B20LP2 & \multicolumn{2}{|c|}{ Lab ID: } & 0905001-17 & & & \\
\hline $15046-84-1$ & Iodine-129 & $<5.05 \mathrm{E}-3$ & $\mathrm{ug} / \mathrm{L}$ & $5.05 \mathrm{E}-3$ & $10 / 06 / 09$ & 9I14001 & PNNL-AGG-415 \\
\hline HEIS No. & B20LR4 & \multicolumn{2}{|c|}{ Lab ID: } & 0905001-18 & & & \\
\hline $15046-84-1$ & Iodine-129 & $7.53 \mathrm{E}-3$ & $\mathrm{ug} / \mathrm{L}$ & $5.05 \mathrm{E}-3$ & $10 / 07 / 09$ & 9I14001 & PNNL-AGG-415 \\
\hline HEIS No. & B20LT1 & \multicolumn{2}{|c|}{ Lab ID: } & 0905001-19 & & & \\
\hline $15046-84-1$ & Iodine-129 & $<5.05 \mathrm{E}-3$ & $\mathrm{ug} / \mathrm{L}$ & $5.05 \mathrm{E}-3$ & $10 / 07 / 09$ & 9I14001 & PNNL-AGG-415 \\
\hline HEIS No. & B20LT4 & \multicolumn{2}{|c|}{ Lab ID: } & 0905001-20 & & & \\
\hline $15046-84-1$ & Iodine-129 & $1.40 \mathrm{E}-2$ & $\mathrm{ug} / \mathrm{L}$ & $1.00 \mathrm{E}-2$ & $9 / 24 / 09$ & 9I14001 & PNNL-AGG-415 \\
\hline HEIS No. & B20MX3 & \multicolumn{2}{|c|}{ Lab ID: } & 0905001-21 & & & \\
\hline $15046-84-1$ & Iodine-129 & $<5.05 \mathrm{E}-3$ & $\mathrm{ug} / \mathrm{L}$ & $5.05 \mathrm{E}-3$ & $10 / 07 / 09$ & 9I14002 & PNNL-AGG-415 \\
\hline HEIS No. & B20PJ5 & \multicolumn{2}{|c|}{ Lab ID: } & $0905001-22$ & & & \\
\hline $15046-84-1$ & Iodine-129 & $1.71 \mathrm{E}-2$ & $\mathrm{ug} / \mathrm{L}$ & $1.00 \mathrm{E}-2$ & $9 / 24 / 09$ & 9I14002 & PNNL-AGG-415 \\
\hline HEIS No. & B20PP7 & & ID: & $0905001-23$ & & & \\
\hline $15046-84-1$ & Iodine-129 & $1.35 \mathrm{E}-2$ & $\mathrm{ug} / \mathrm{L}$ & $1.00 \mathrm{E}-2$ & $9 / 24 / 09$ & 9I14002 & PNNL-AGG-415 \\
\hline
\end{tabular}


Radionuclides By ICP-MS

\begin{tabular}{|c|c|c|c|c|c|c|c|}
\hline CAS \# & Analyte & Results & Units & EQL & Analyzed & Batch & Method \\
\hline HEIS No. & B20P35 & \multicolumn{2}{|c|}{ Lab ID: } & 0905001-24 & & & \\
\hline $15046-84-1$ & Iodine-129 & $1.79 \mathrm{E}-2$ & $\mathrm{ug} / \mathrm{L}$ & $1.00 \mathrm{E}-2$ & $9 / 24 / 09$ & 9I14002 & PNNL-AGG-415 \\
\hline HEIS No. & B20NX6 & \multicolumn{2}{|c|}{ Lab ID: } & 0905001-25 & & & \\
\hline $15046-84-1$ & Iodine-129 & $1.35 \mathrm{E}-2$ & $\mathrm{ug} / \mathrm{L}$ & $1.00 \mathrm{E}-2$ & $9 / 24 / 09$ & 9I14002 & PNNL-AGG-415 \\
\hline HEIS No. & B20MY5 & \multicolumn{2}{|c|}{ Lab ID: } & 0905001-26 & & & \\
\hline $15046-84-1$ & Iodine-129 & $<5.05 \mathrm{E}-3$ & $\mathrm{ug} / \mathrm{L}$ & $5.05 \mathrm{E}-3$ & $10 / 07 / 09$ & 9I14002 & PNNL-AGG-415 \\
\hline
\end{tabular}




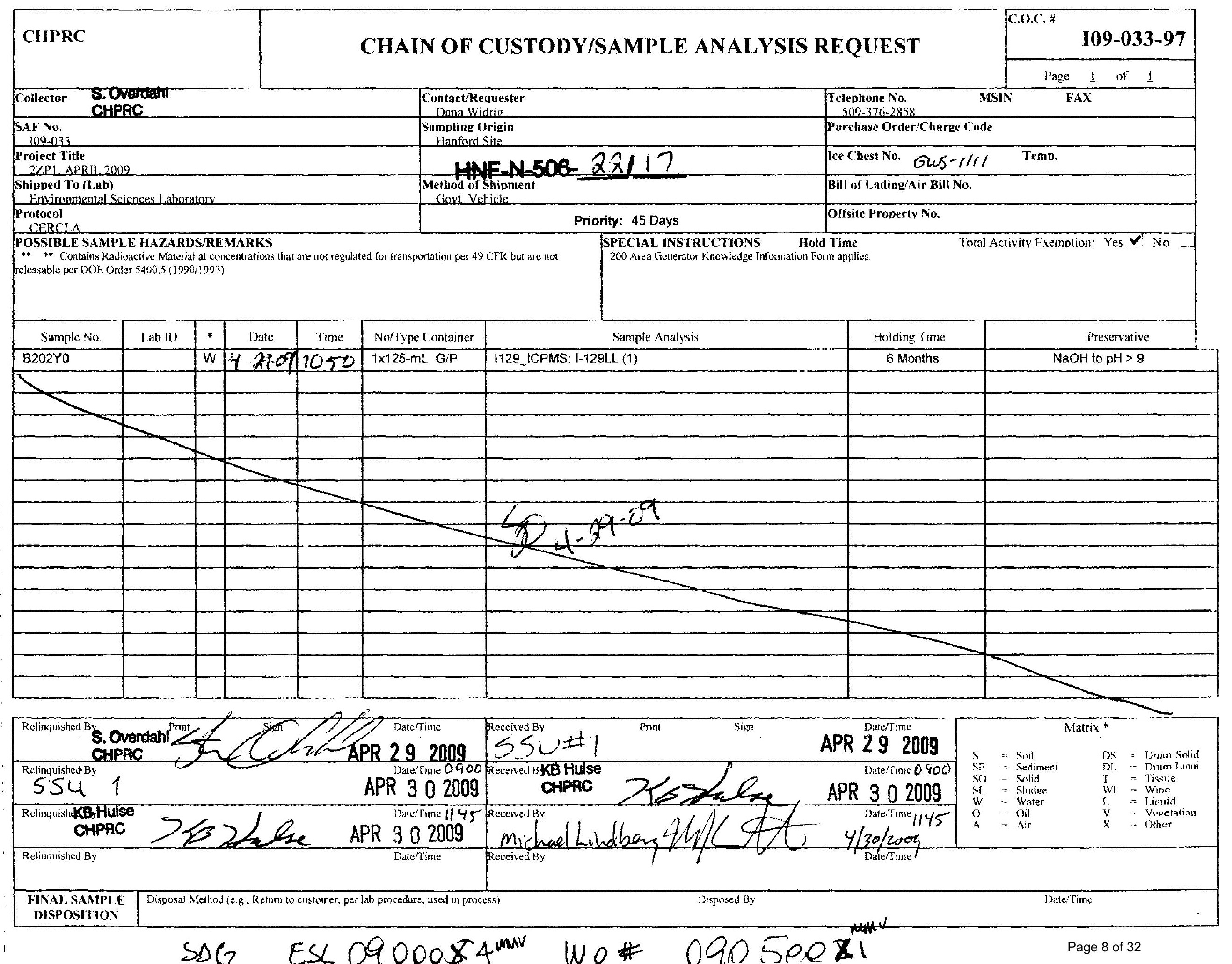




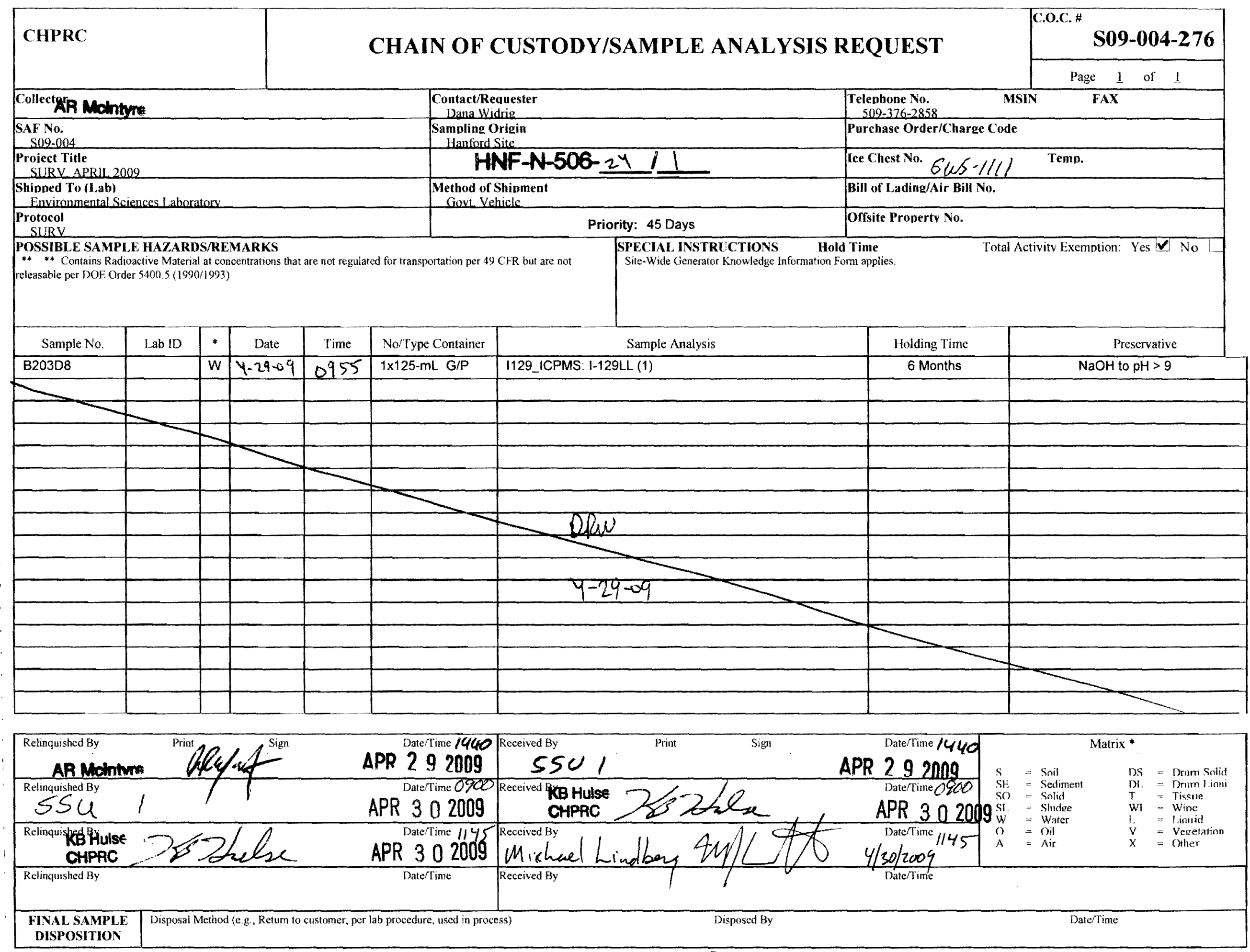

$$
\text { SOG ESL } 090004 \quad W_{0}=0905001
$$




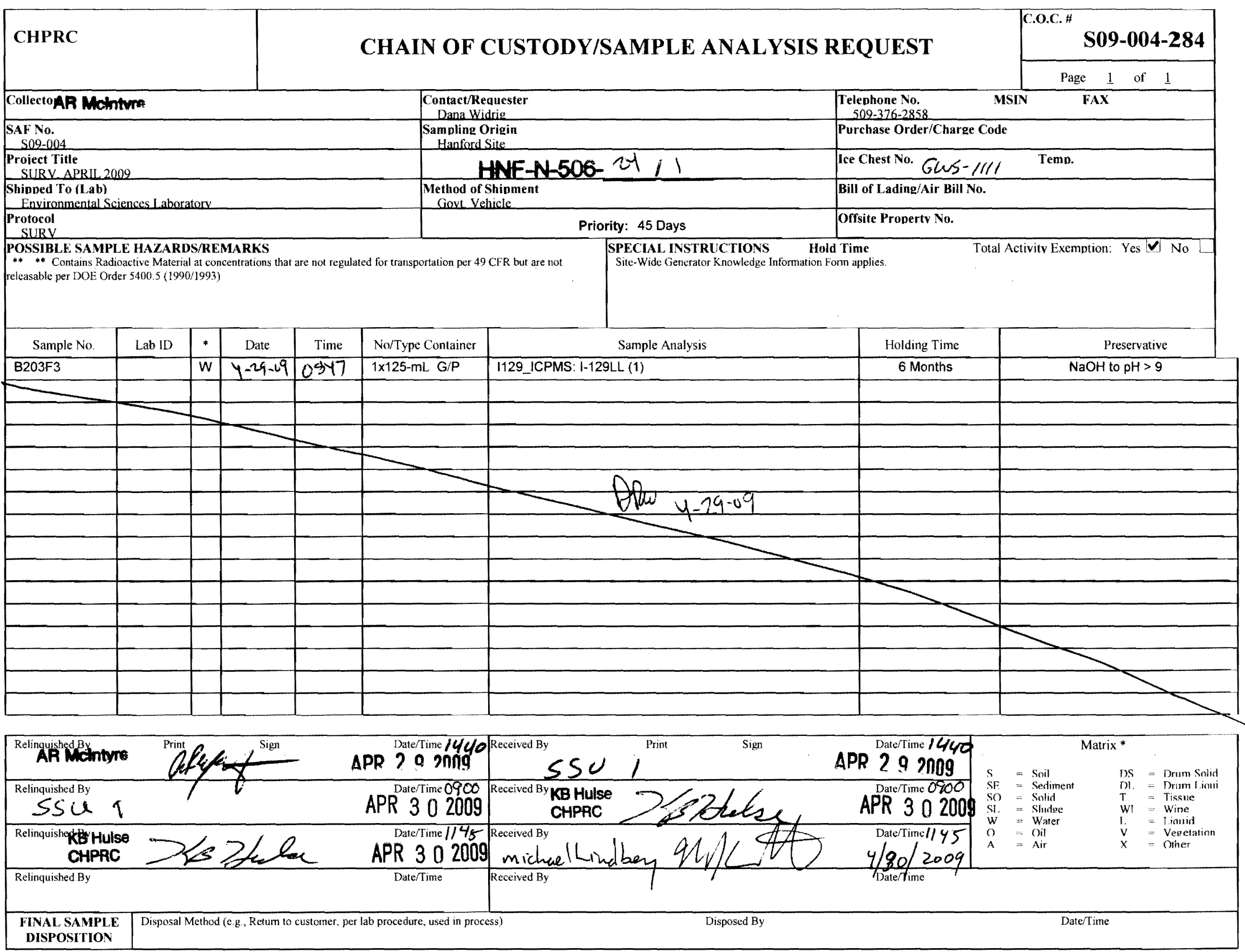




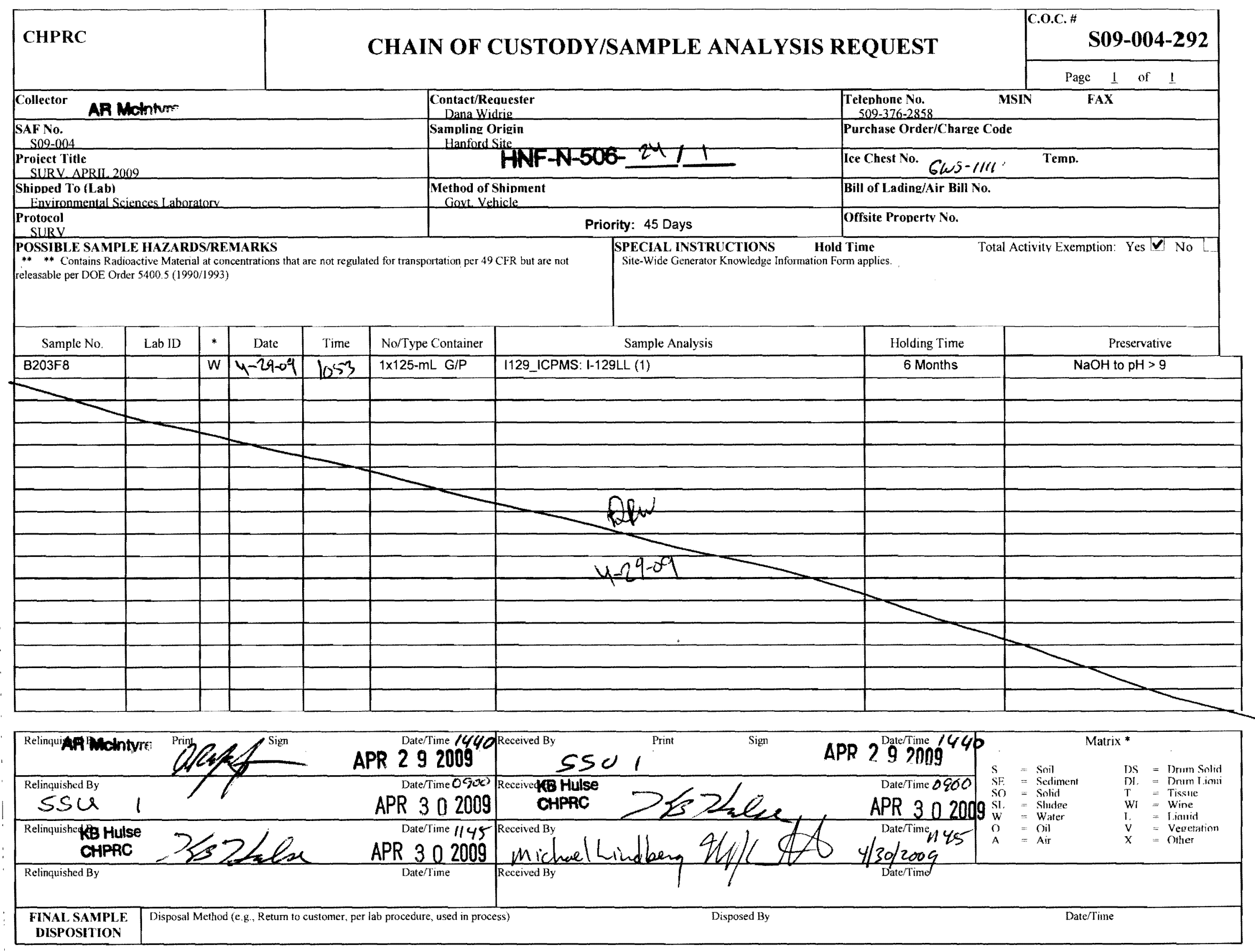




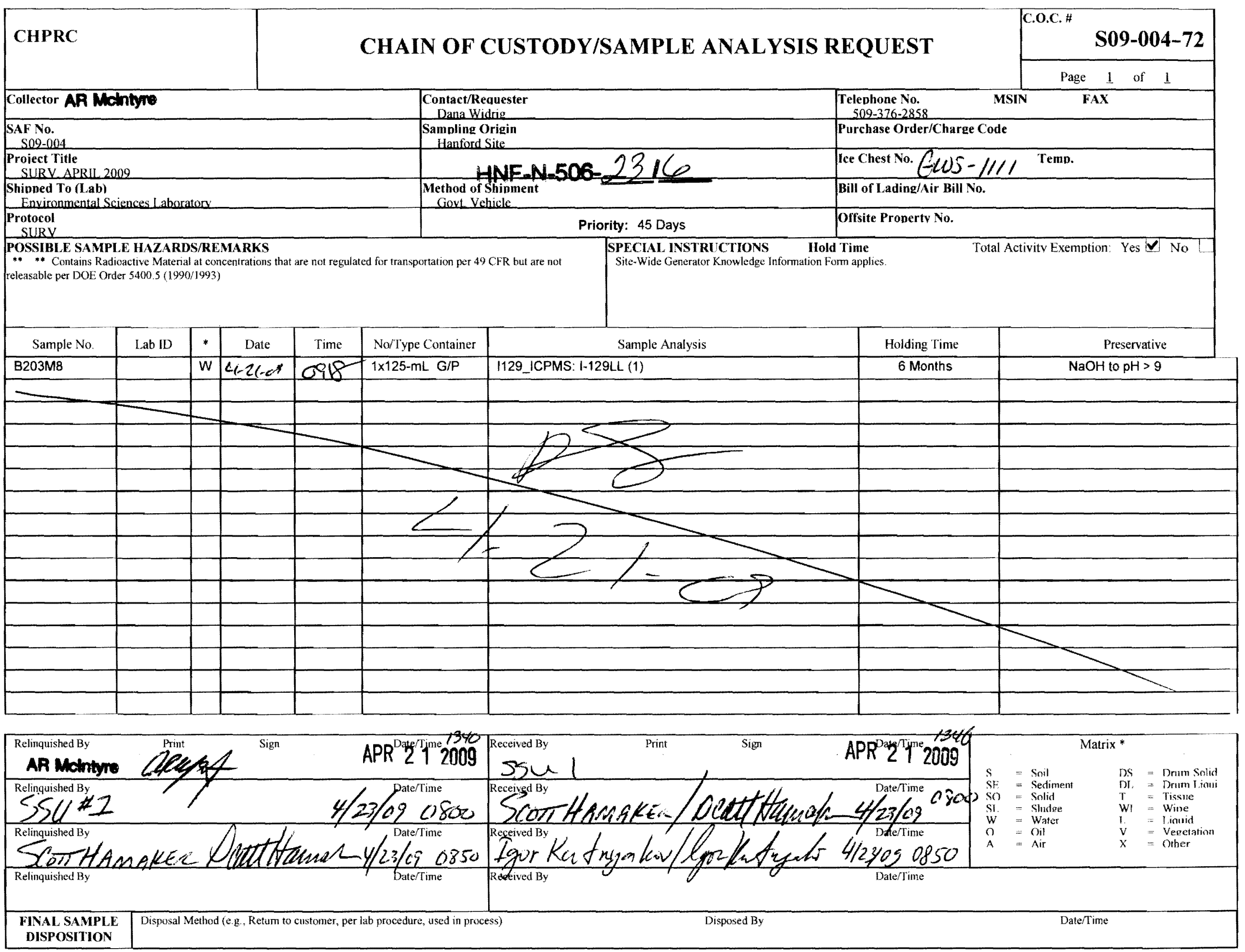




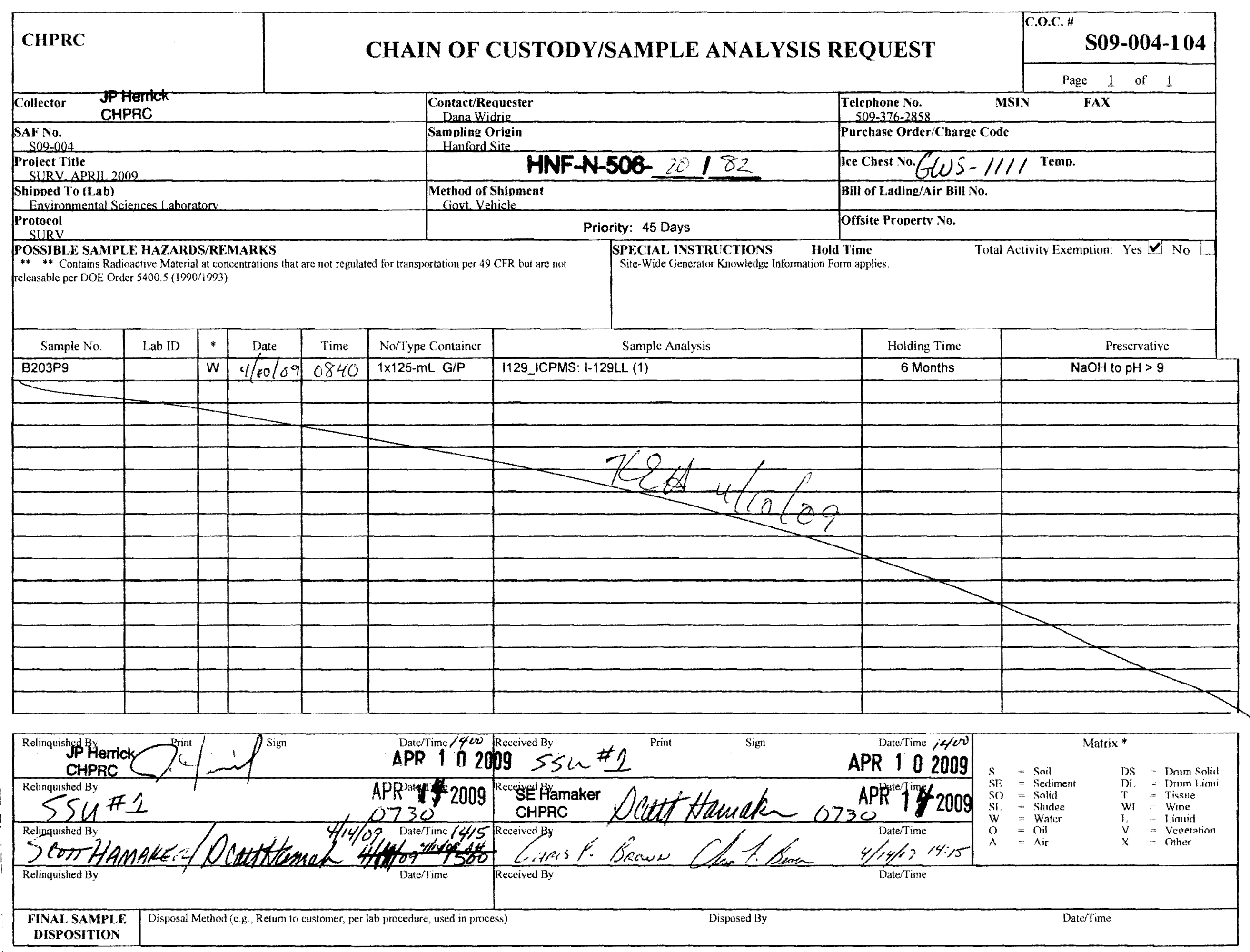




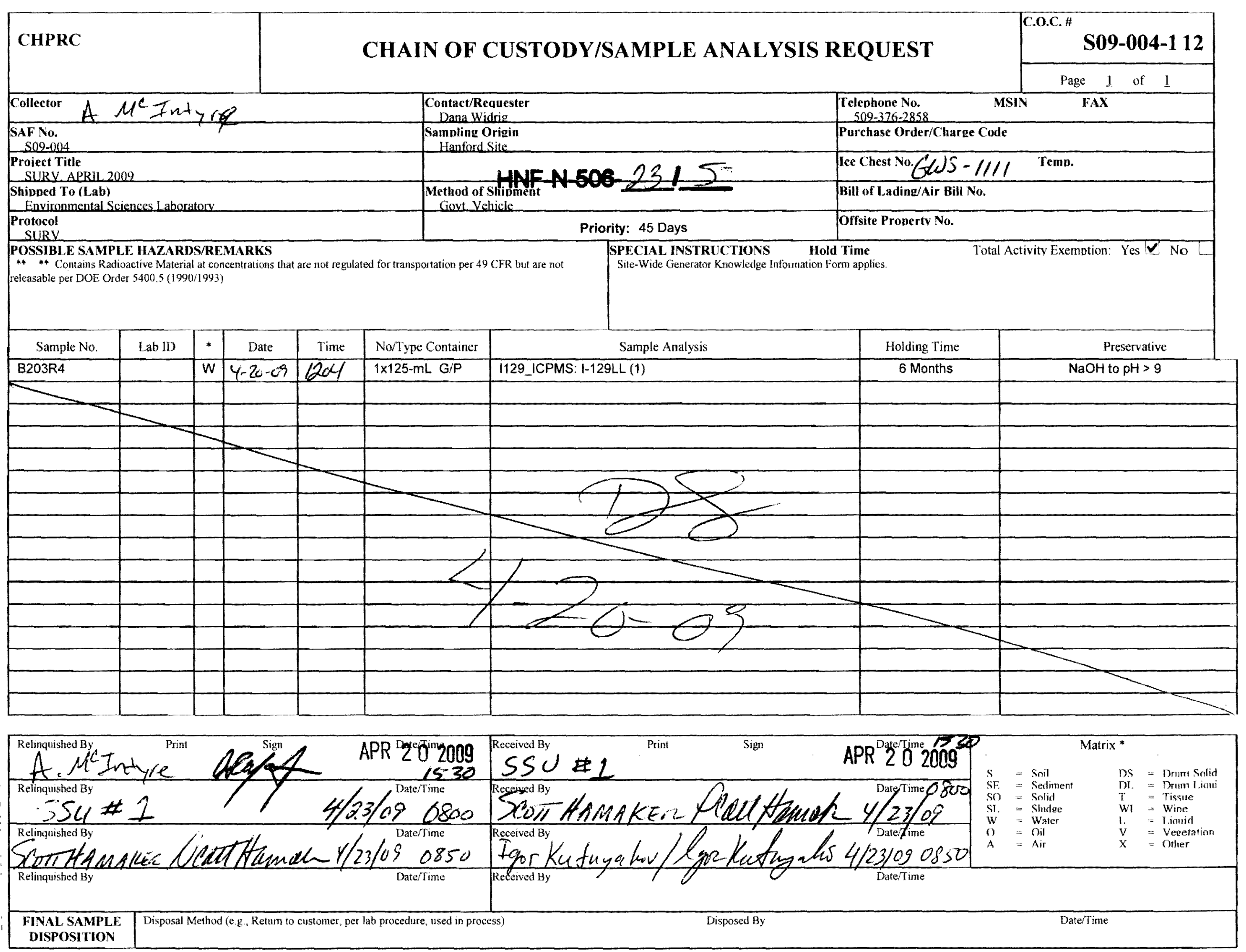




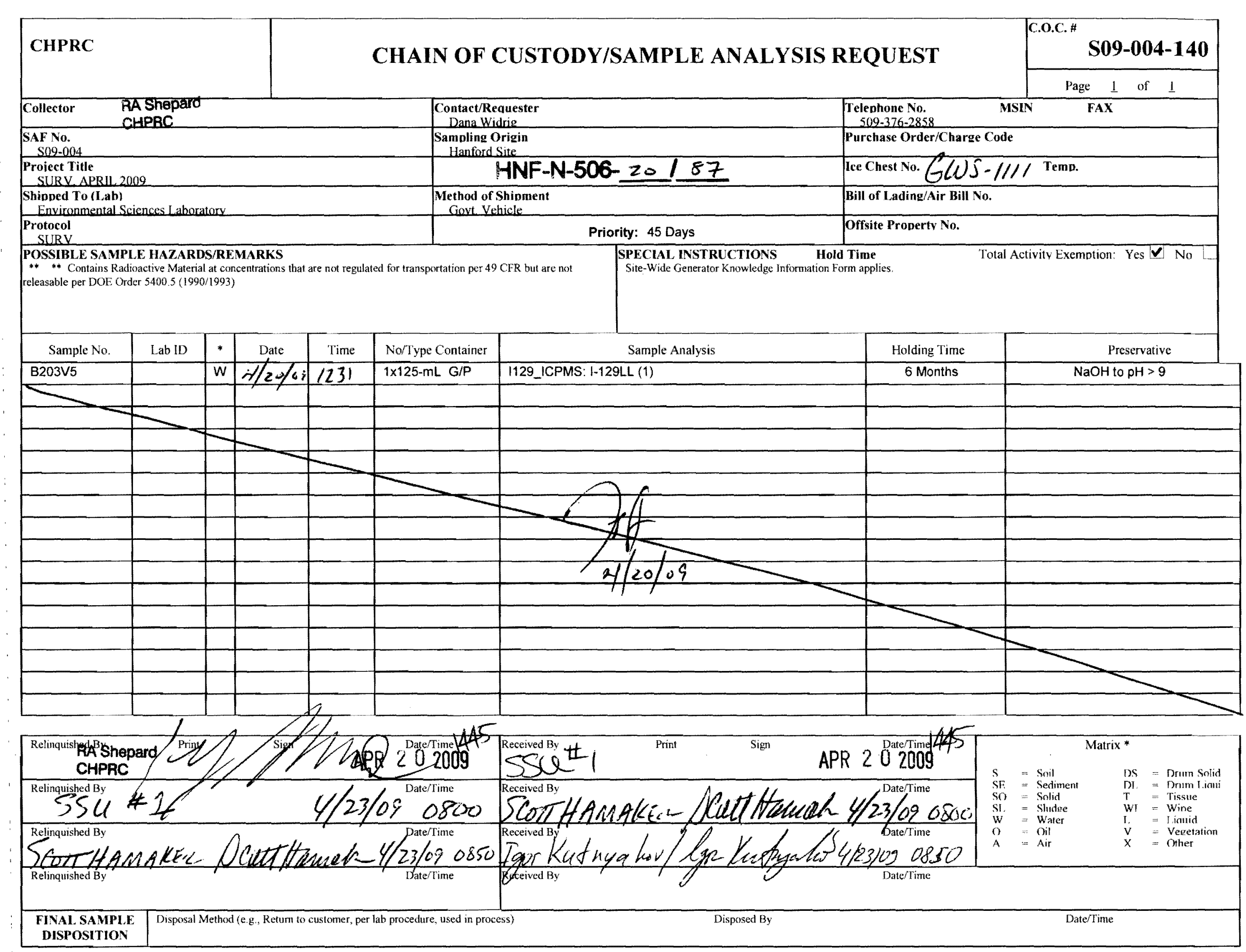




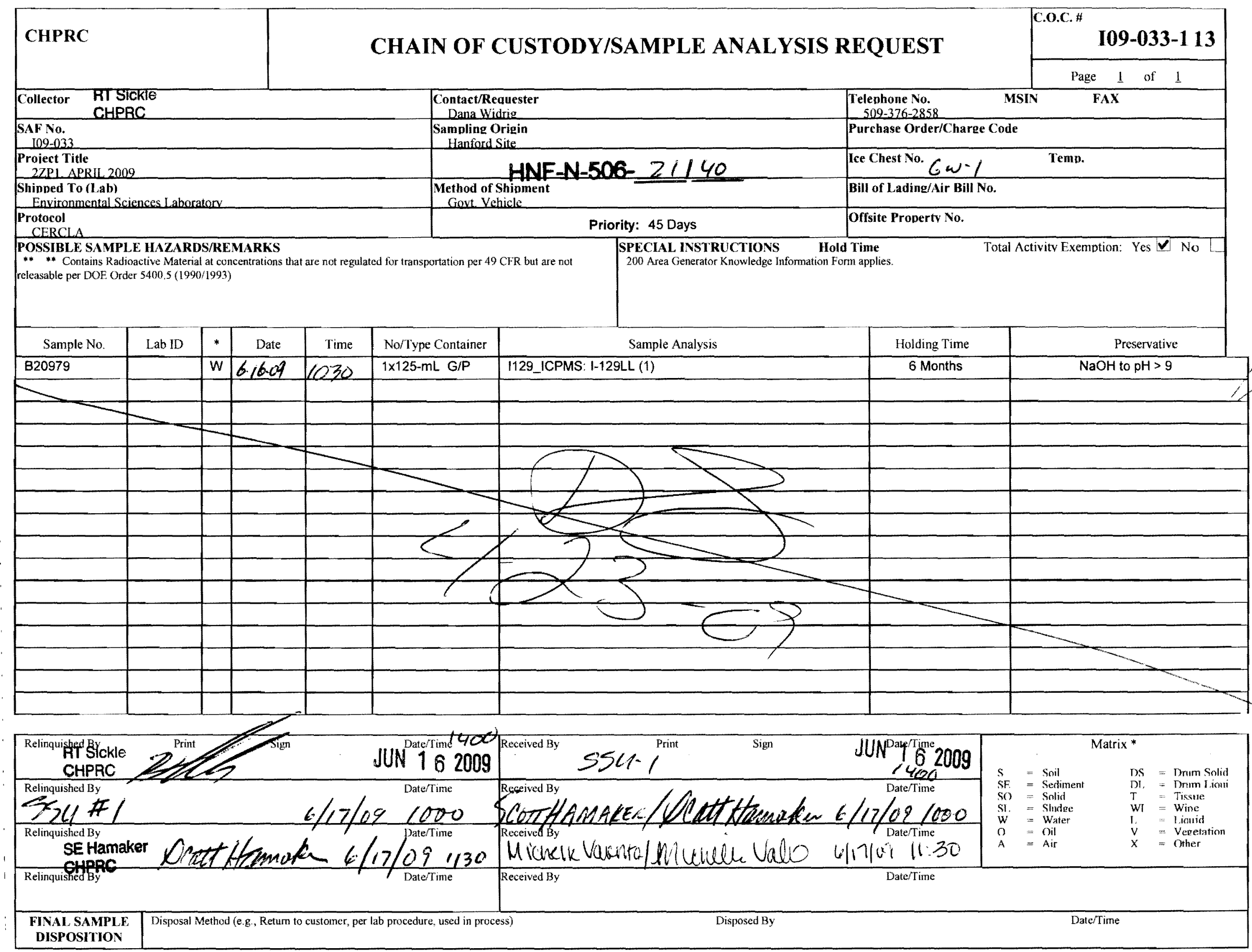




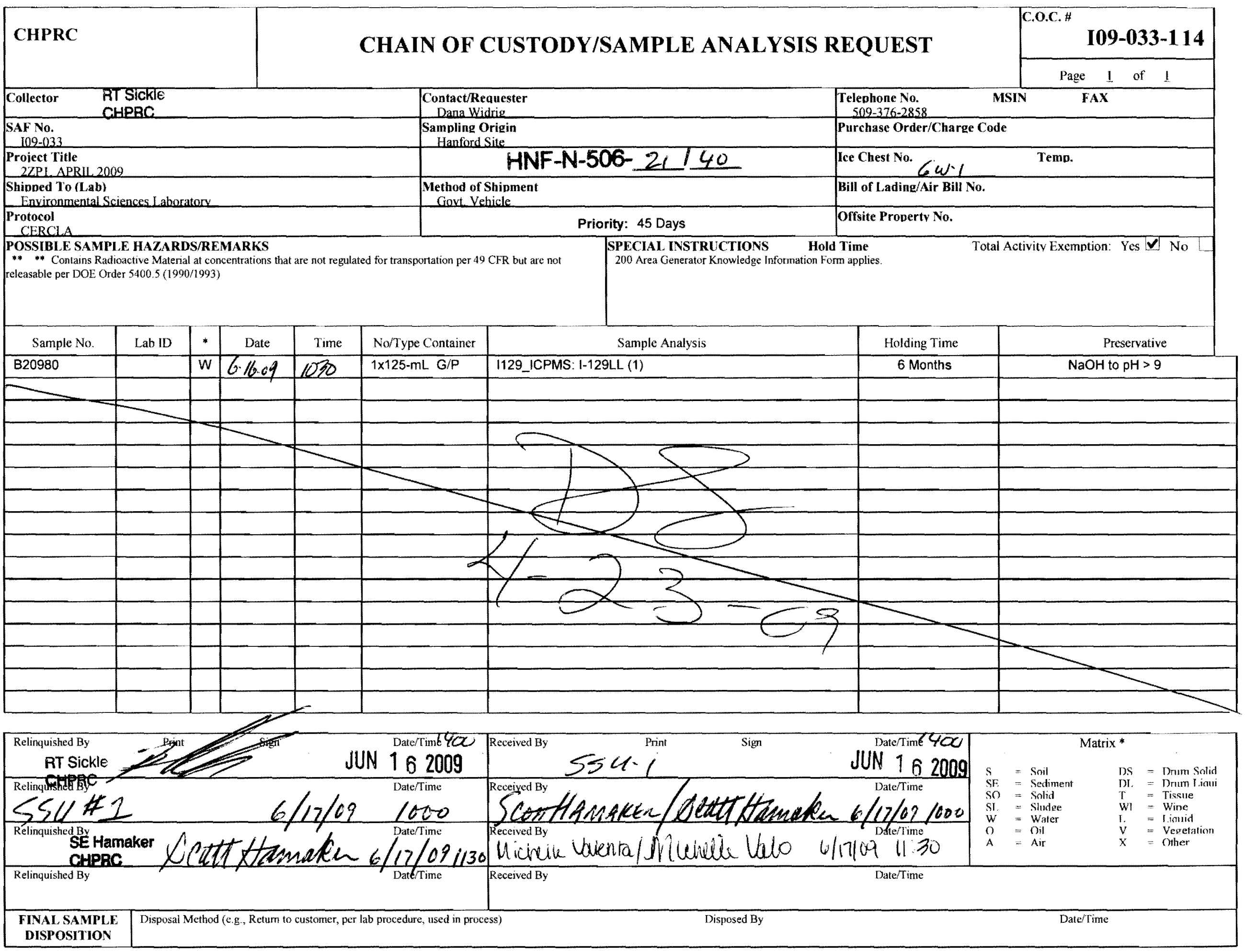




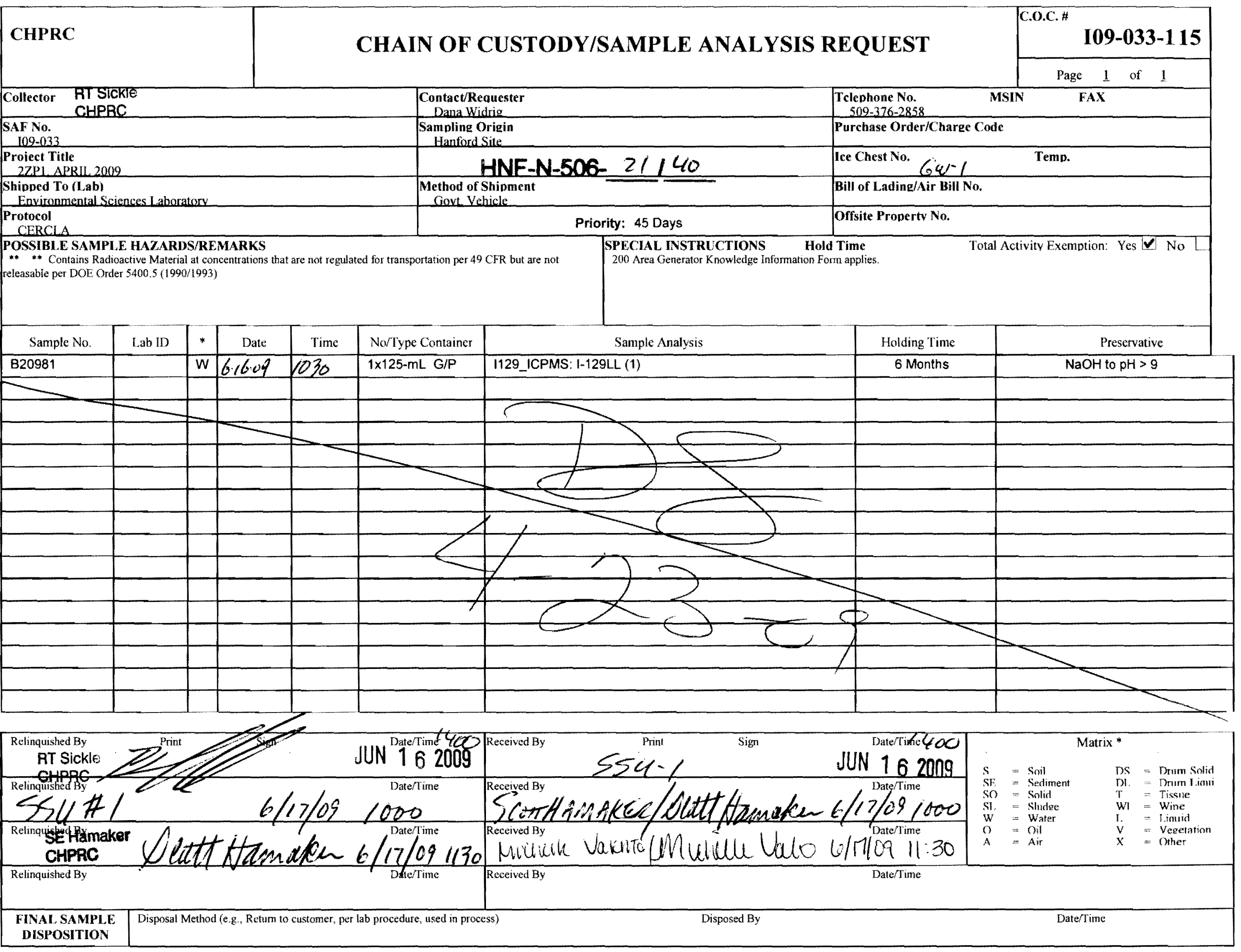




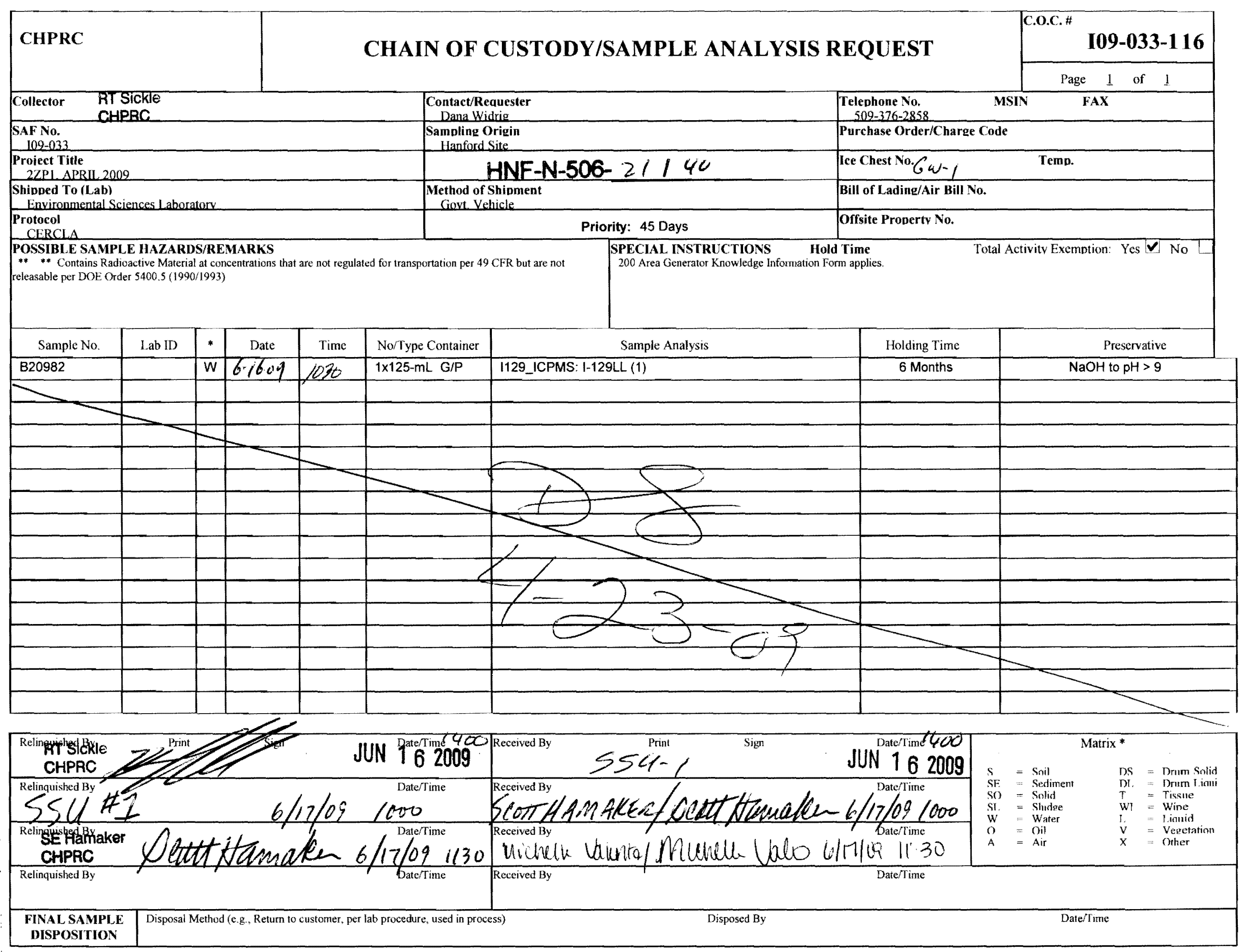




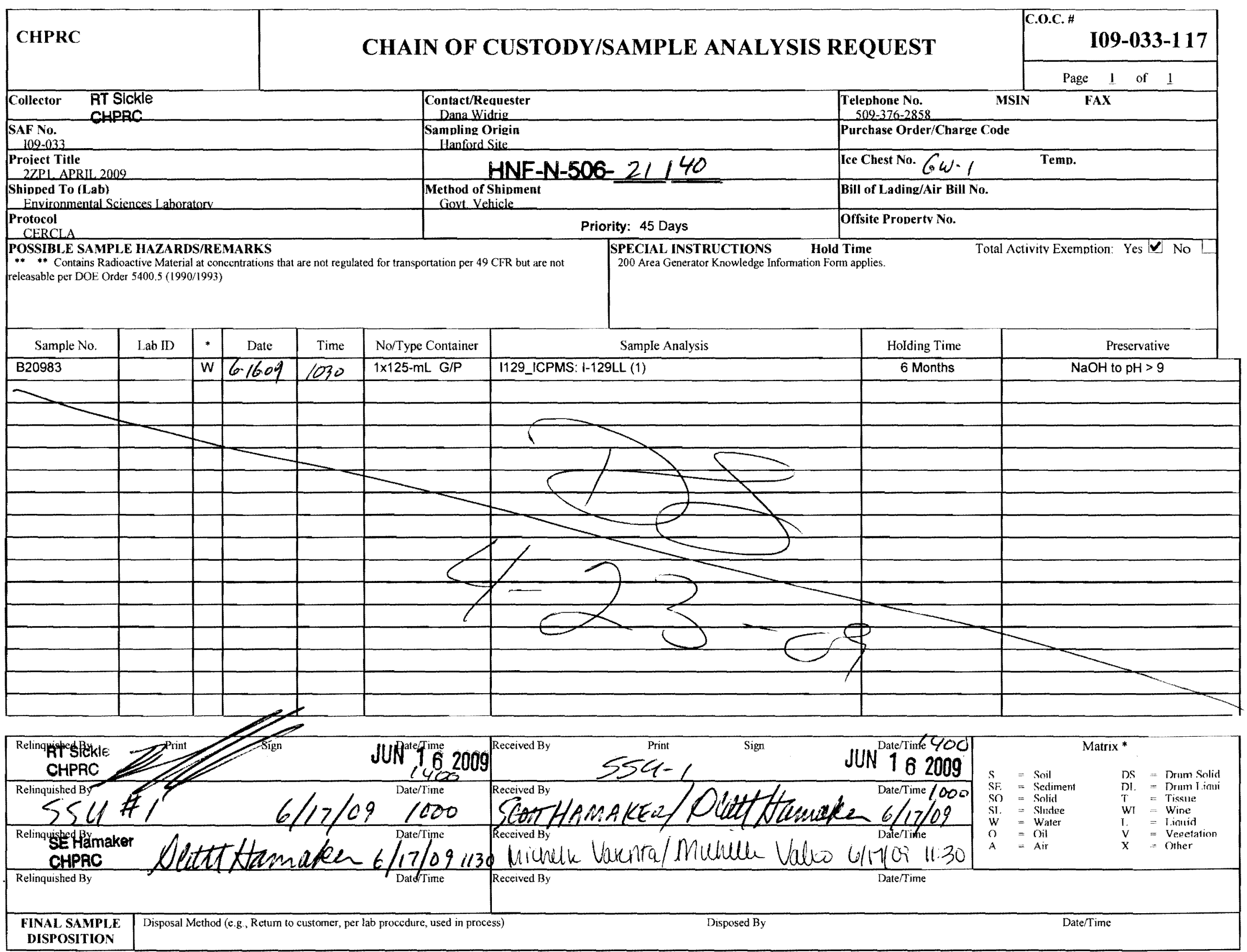




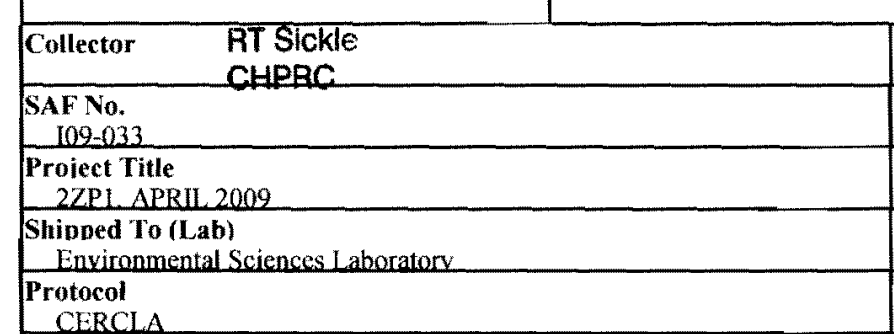

Contact/Requester

Dana Widrie

Sampling Origin

Hanford Site

\section{HNF-N-506-21140}

Method of Shioment Gout Vehicle
Teleohone No.

509-376-2858 Purchase Order/Charge Code

Ice Chest No. CW. Temp.

Bill of Lading/Air Bill No.

Offsite Property No.

POSSIBLE SAMPLE HAZARDS/REMARKS

Priority: 45 Days

** ** Contains Radioactive Material at concentrations that are not regulated for transportation per 49 CFR but are not

SPECIAL INSTRUCTIONS Hold Time

200 Area Generator Knowledge Information Form applies

Total Activity Exemption: Yes $\underline{\square}$ No

eleasable per DOE Order 5400.5 (1990/1993)

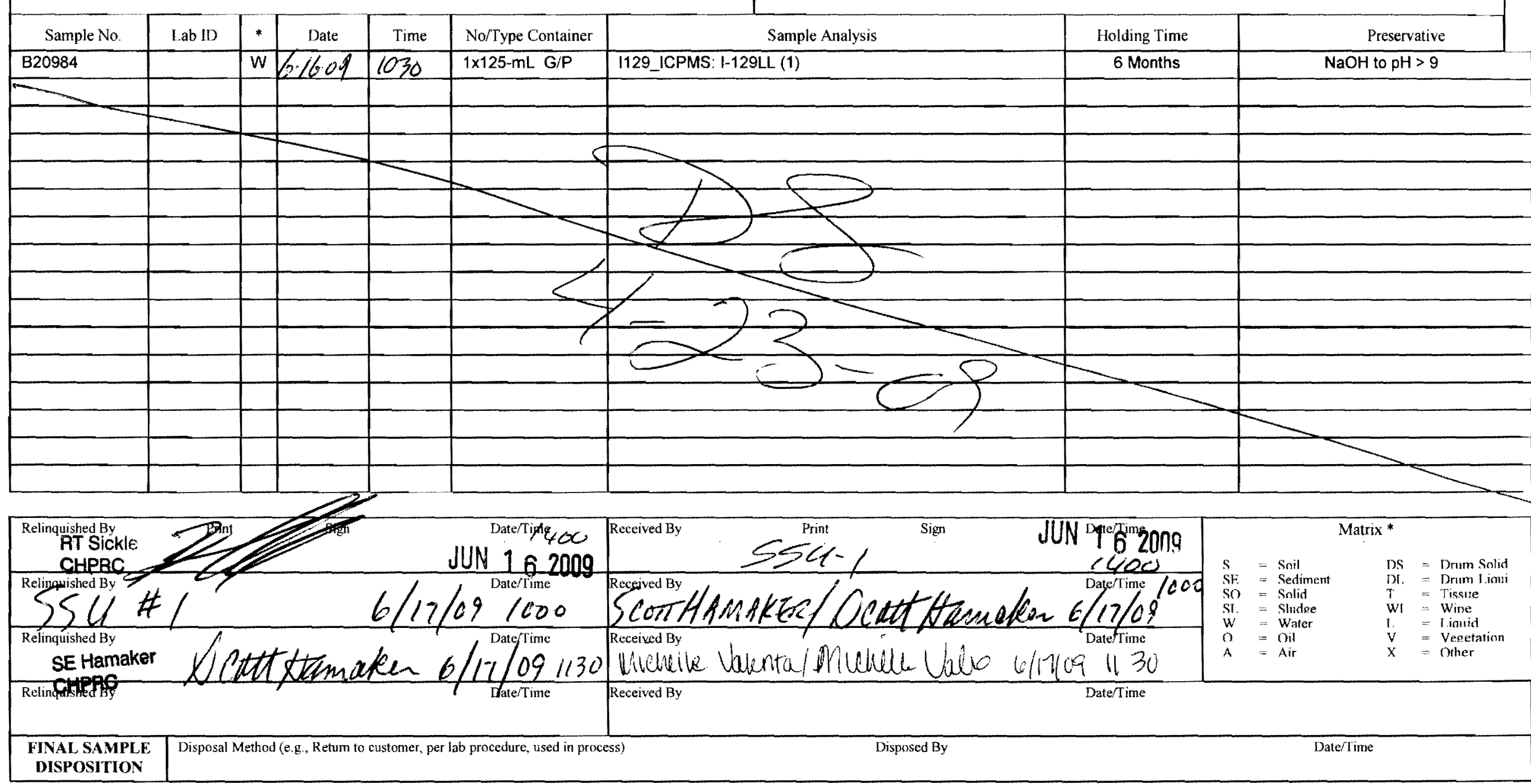




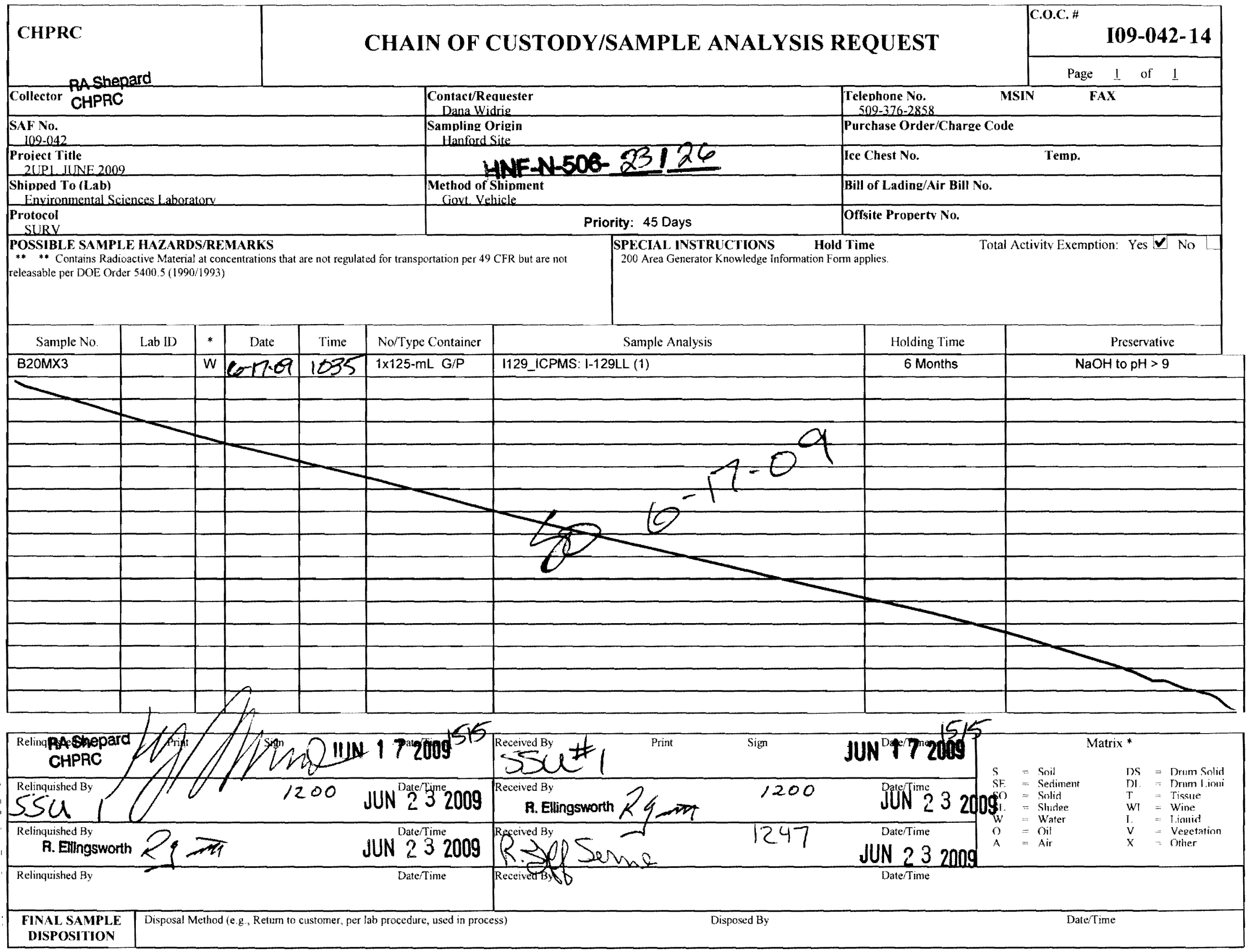

Page 24 of 32 
LLWMACII-BA JUNE 2009

Shipned To (Lab)

Environmental Sciences Laboratory

Protocol

POSSIBLE SAMPIE HAZARDS/REMAKKS

POSSIBLE SAMPCE HAZARDS/REMARKS
$*_{* *}$ Contains Radioactive Material at concentrations that are not regulated for transpotation per 49 CFR but are not releasable per DOE Order 5400.5 (1990/1993)

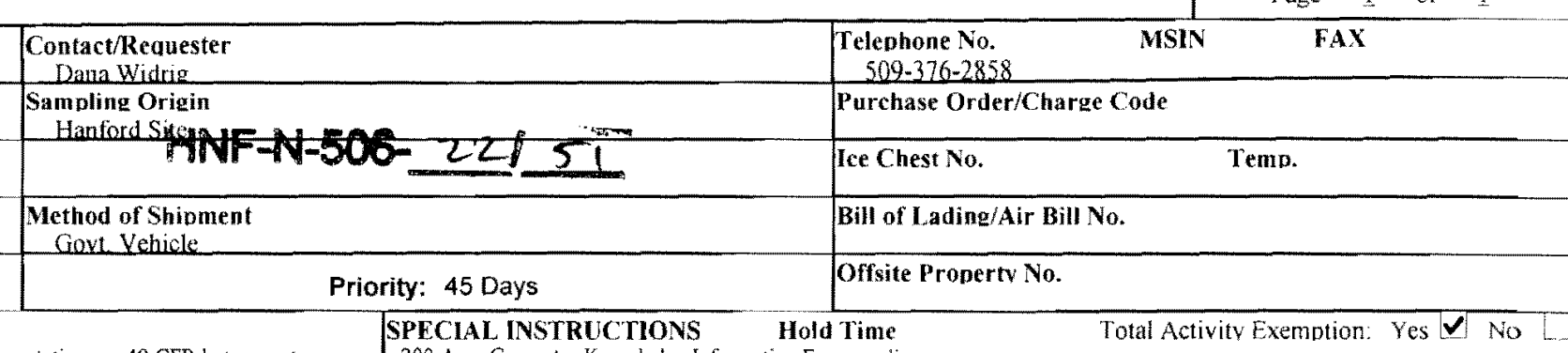

SPECIAL INSTRUCTIONS Hold Time
200 Area Generator Krowledge Information Form applie

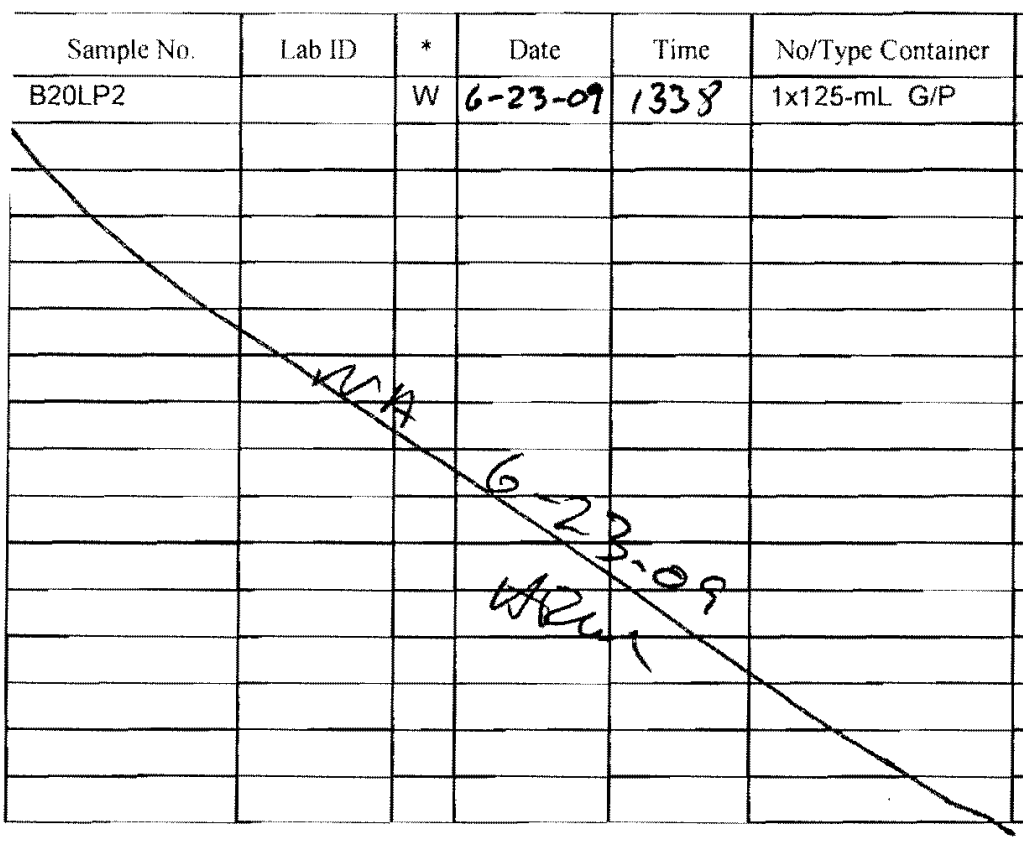

\begin{tabular}{|c|c|c|}
\hline Sample Analysis & Holding Time & Preservative \\
\hline 1129_ICPMS: I-129LL (1) & 6 Months & $\mathrm{NaOH}$ to $\mathrm{pH}>9$ \\
\hline & & \\
\hline & & \\
\hline & & \\
\hline & & \\
\hline & & \\
\hline & & \\
\hline & & \\
\hline & & \\
\hline & & \\
\hline & & \\
\hline & & \\
\hline & & \\
\hline & & \\
\hline & & \\
\hline & & \\
\hline
\end{tabular}
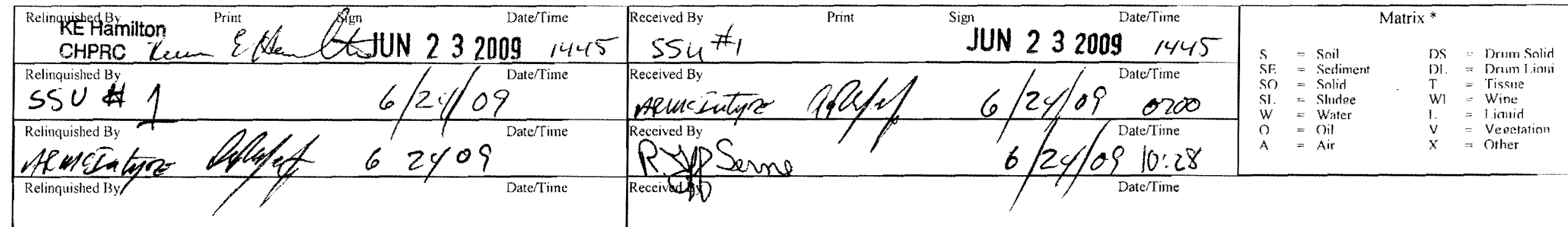

FINAL SAMPLE

DISPOSITION 


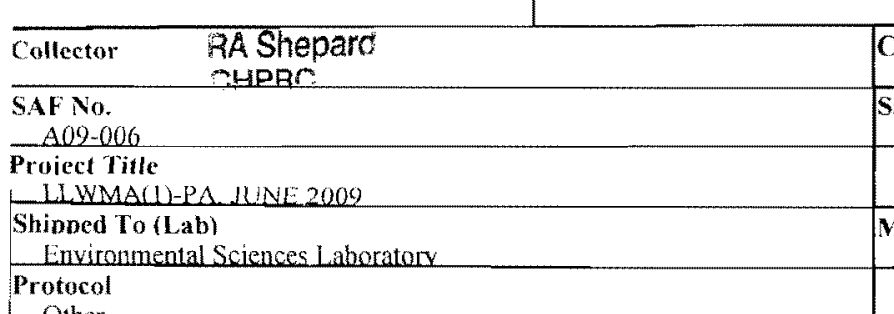

Contact/Requester

Dana Widrie

Sampling Origin

\section{ANE-N-506-21/43}

Method of Shinmen

Govt. Vehicle
Telephone No.

$509-376-2858$

Purchase Order/Charge Code

Ice Chestifom -33 Temp.

Bill of Lading/Air Bill No.

Offsite Propertv No.

Priority: 45 Days

SPECIAL INSTRUCTIONS Hold Time

200 Area Generator Knowledge Information Form applies

ESL - Hold all samples until all 10 are received and run the complete set at ONE tine

** ** Contains Radicactive Material at concentrations that are not regulated for transportation per $49 \mathrm{CER}$ but are not reteasable per DOE Order 5400.5 (1990/1993)

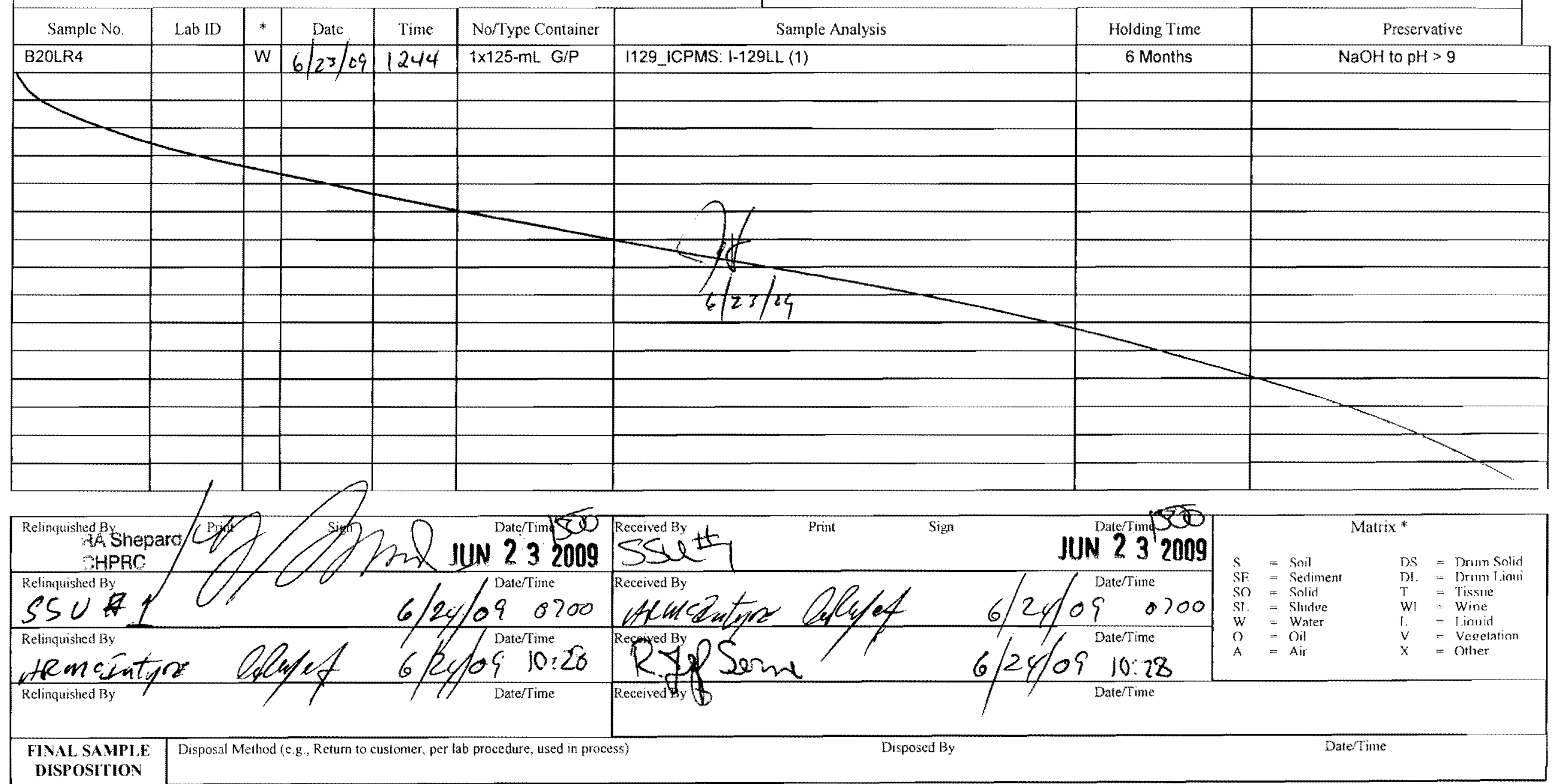




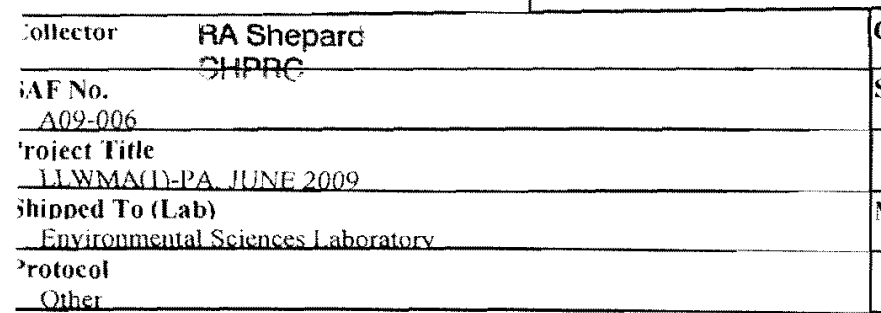

\begin{tabular}{l}
$\begin{array}{c}\text { Contact/Requester } \\
\text { Dana Widrig }\end{array}$ \\
$\begin{array}{c}\text { Sampling Origin } \\
\text { Hanford Site }\end{array}$ \\
\hline $\begin{array}{c}\text { Method of Shipment } \\
\text { Gove. Yehicle }\end{array}$ \\
\hline
\end{tabular}

\begin{tabular}{|c|c|}
\hline & Page 1 of 1 \\
\hline $\begin{array}{cc}\text { Telephone No. } & \text { MSIN } \\
509-376-2858 & \\
\end{array}$ & FAX \\
\hline \multicolumn{2}{|l|}{ Purchase Order/Charge Code } \\
\hline Ice chestsm $51-313$ & Temp. \\
\hline Bill of Lading/Air Bill No. & \\
\hline
\end{tabular}

POSSIBLE SAMPLE IAZARDS/REMARKS

SPECIAL INSTRUCTIONS Hold Time

Total Activitv Exemption: Yes $\checkmark$ No

** * Contains Radivactive Material at concentrations that are not regulated for transportation per 49 CFR but are not 200 Area Generator Knowledge Information Form applies

eleasabie per DOE Order 5400.5 (1900/1993)

ESL. - Hold all samples until all 10 are reccived and run the complete set at ONE time

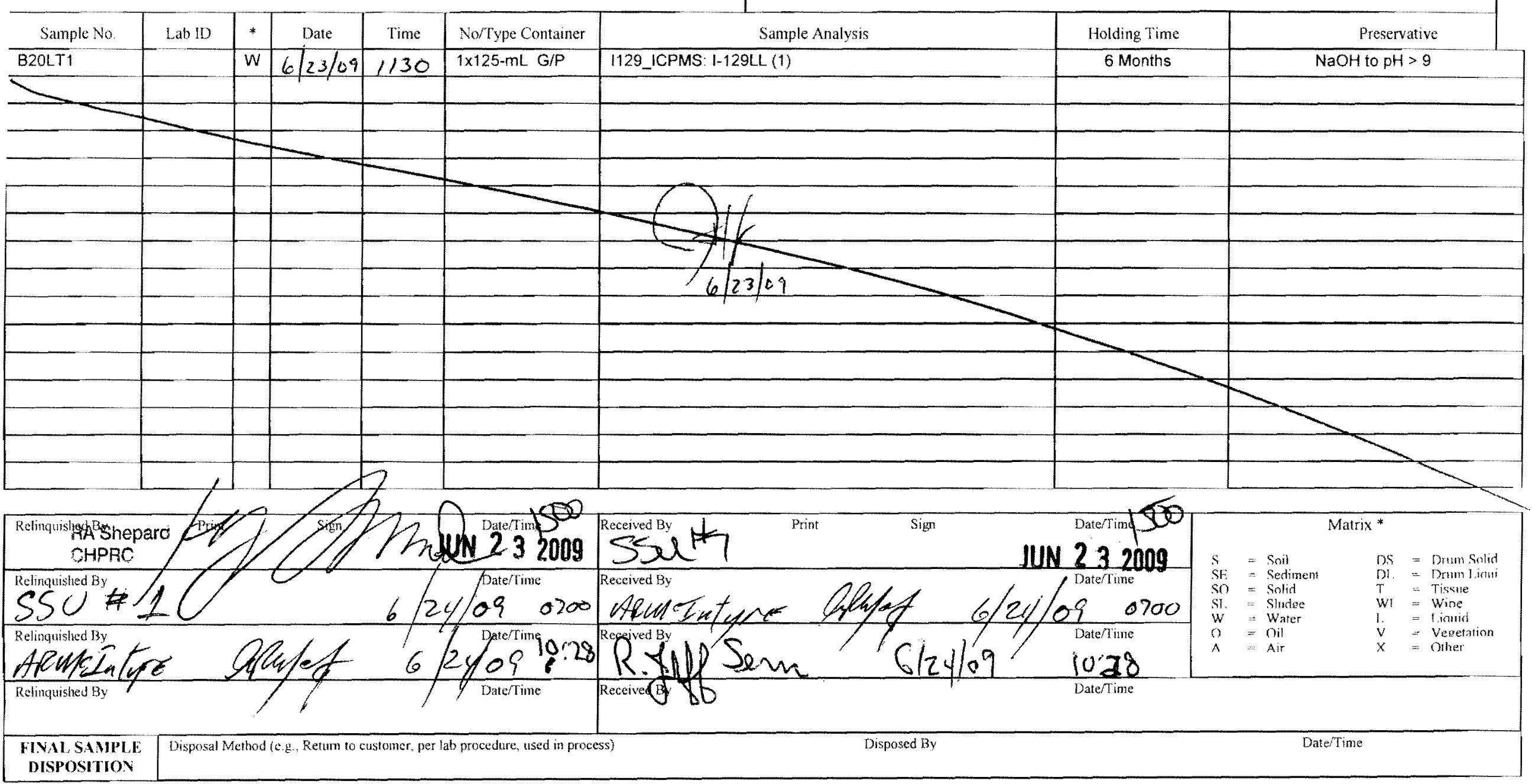




\section{allector RA Sheparo}

iAf No.

IMPAC

A09-006

roiect Title

ILWMACL-BA LUNL2000

ihipped To (lab)

Envirommental Sciences Labocator

rotocol

$\frac{\text { Other }}{\text { OSSIBLE SAMPLE HAZARDS/REMARKS }}$

**** Contains Radioactive Material at concentrations that are not regulated for transportation per 49 CFR but are not

Contact/Requester

Dampling Origin

Sampling Origin
Hanford Site

Method of Shiomen

$\begin{aligned} & \text { Telephone No. } \\ & 509-376-2858\end{aligned}$
Purchase Order/Charge Code

Ice chestignc Temb.

Goxt Vehicle Bill of Lading/Air Bill No.

eleasable per DOE Order 5400.5 (1990/1993

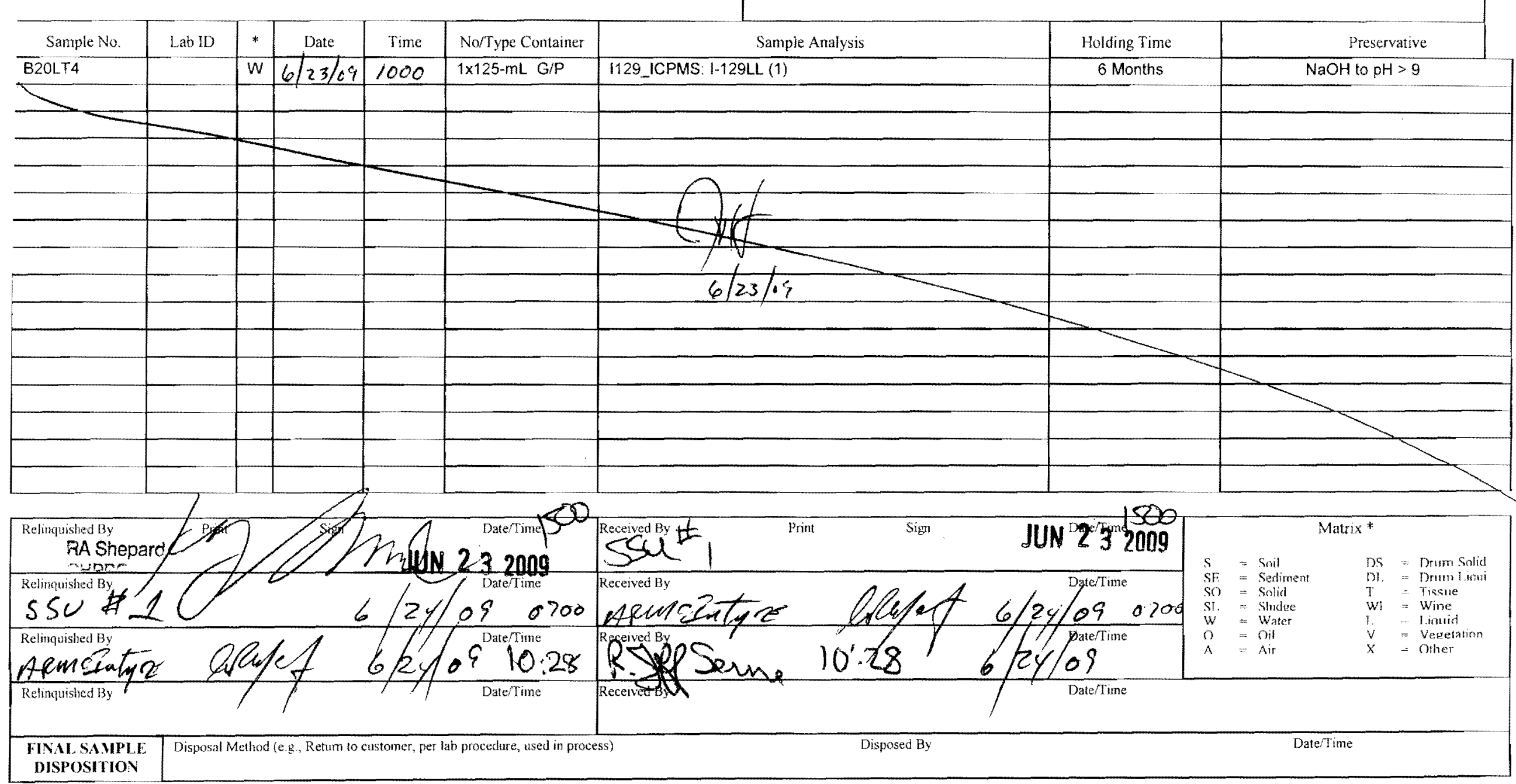




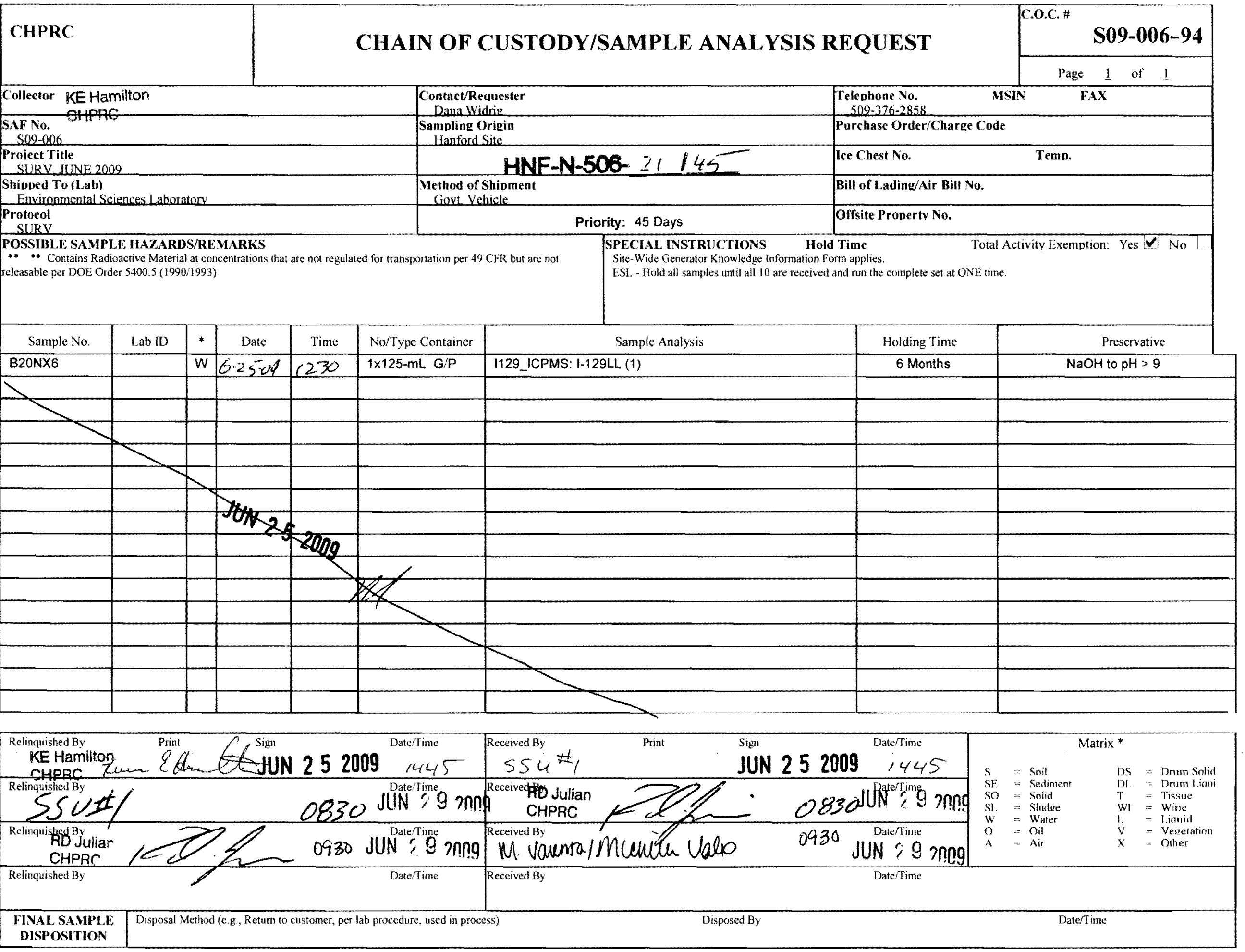




\begin{tabular}{|l|}
\hline Collector PB Cano \\
\multicolumn{1}{|c|}{ CHPRC } \\
\hline SAF No. \\
S09-006 \\
\hline $\begin{array}{l}\text { Proiect Title } \\
\text { SURV JUNE 2009 }\end{array}$ \\
\hline $\begin{array}{l}\text { Shipped To (Lab) } \\
\text { Environmental Sciences Laboratory } \\
\text { Protocol }\end{array}$ \\
\hline SURY
\end{tabular}

\begin{tabular}{l}
$\begin{array}{l}\text { Contact/Requester } \\
\text { Dana Widrig }\end{array}$ \\
$\begin{array}{l}\text { Sampling Origin } \\
\text { Hanford Site }\end{array}$ \\
$\begin{array}{l}\text { Method of Shipment } \\
\text { Govt Vehicle }\end{array}$ \\
\hline$\quad$ Priority: 45 Days
\end{tabular}

\begin{tabular}{|l|l|}
\hline $\begin{array}{l}\text { Telephone No. } \\
509-376-2858\end{array}$ \\
\hline Purchase Order/Charge Code \\
\hline Ice Chest No. \\
\hline Bill of Lading/Air Bill No. \\
\hline Offsite Property No.
\end{tabular}

SPECIAL INSTRUCTIONS Hold Time

Site-Wide Generator Knowledge Information Form applies.

Total Activity Exemption: Yes $\boldsymbol{v}$ No

OSSIBLE SAMPLE HAZARDS/REMARKS

** Contains Radioactive Material at concentrations that are not regulated for transportation per $49 \mathrm{CFR}$ but are not

ESL - Hold all samples until all 10 are received and run the complete set at ONE time.

\begin{tabular}{|c|c|c|c|c|c|c|c|c|}
\hline Sample No. & Lab ID & $*$ & Date & Time & No/Type Container & Sample Analysis & Holding Time & Preservative \\
\hline B20P35 & & $W$ & $6 \longdiv { 2 6 9 }$ & 1126 & $1 \times 125-\mathrm{mL} \mathrm{G/P}$ & I129_ICPMS: I-129LL (1) & 6 Months & $\mathrm{NaOH}$ to $\mathrm{pH}>9$ \\
\hline 3 & & & & & & & & \\
\hline & & & & & & & & \\
\hline & & 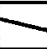 & & & & & & \\
\hline & & & & & & & & \\
\hline & & & & & & & & \\
\hline & & & & & & & & \\
\hline & & & & & & & & \\
\hline & & & & & & & & \\
\hline & & & & & & & & \\
\hline & & & & & & & & \\
\hline & & & & & & & $\gamma$ & \\
\hline & & & & & & & & \\
\hline & & & & & & & & \\
\hline & & & & & & & & \\
\hline & & & & & & & & \\
\hline
\end{tabular}

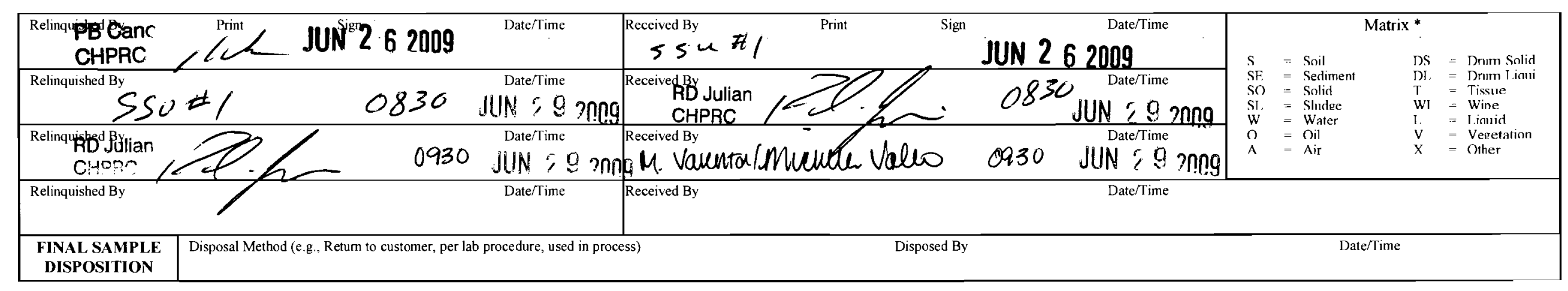


